\title{
Numerical Analysis of Paraffin-wax/Oxygen Hybrid Rocket Engines
}

\author{
Mario Tindaro Migliorino*, Daniele Bianchi ${ }^{\dagger}$, and Francesco Nasuti ${ }^{\ddagger}$ \\ Sapienza University of Rome, 00184 Rome, Italy
}

\begin{abstract}
A predictive numerical approach, based on Reynolds-averaged Navier-Stokes simulations including the effects of turbulence, chemistry, fluid-surface interaction, and radiation has been developed for paraffin-wax/oxygen hybrid rocket engines. A recently fired single-port paraffinbased hybrid rocket engine, with chamber pressures up to 19.1 bar, is taken as reference for the discussion of the results of the numerical simulations, which outline important features of the internal ballistics otherwise not observed in the experiments. For the engine under consideration, radiation accounts for $33 \%-62 \%$ of the total wall heat flux on the grain, depending on the radial dimension and chamber pressure. The rebuilding of the experimental time-averaged regression rate and chamber pressure is carried out with fair success, enabling their prediction with a maximum error of $15 \%$ and $10 \%$, respectively. The numerical model can aid the design and the optimization of future paraffin-based hybrid rocket engines. F $^{*}$
\end{abstract}

\section{Nomenclature}

$t \quad=$ time, $\mathrm{s}$

$N \quad=$ number of species

$\mathbf{v}=$ flow velocity, $\mathrm{m} / \mathrm{s}$

$p \quad=$ pressure, $\mathrm{Pa}$

$T=$ temperature, $\mathrm{K}$

$\rho \quad=\quad$ density, $\mathrm{kg} / \mathrm{m}^{3}$

$h=$ enthalpy per unit mass, $\mathrm{J} / \mathrm{kg}$

$q=$ heat flux, $\mathrm{W} / \mathrm{m}^{2}$

$k=$ thermal conductivity, $\mathrm{W} /(\mathrm{m} \cdot \mathrm{K})$

$\mu \quad=\quad$ dynamic viscosity, $\mathrm{Pa} \cdot \mathrm{s}$

$\mathrm{O} / \mathrm{F}=$ oxidizer-to-fuel ratio

* Research Fellow, Dipartimento di Ingegneria Meccanica e Aerospaziale, via Eudossiana 18, Member AIAA, corresponding author, mariotindaro.migliorino@uniroma1.it.

$\dagger$ Assistant Professor, Dipartimento di Ingegneria Meccanica e Aerospaziale, via Eudossiana 18, Member AIAA.

$\doteqdot$ Associate Professor, Dipartimento di Ingegneria Meccanica e Aerospaziale, via Eudossiana 18, Associate Fellow AIAA.

*Presented as Paper 2019-4261 at the AIAA Propulsion and Energy 2019 Forum, Indianapolis, IN, 19-22 August 2019 


$$
\begin{aligned}
& y=\text { mass fraction } \\
& c_{p} \quad=\quad \text { specific isobaric heat, } \mathrm{J} /(\mathrm{kg} \cdot \mathrm{K}) \\
& c \quad=\quad \text { specific heat, } \mathrm{J} /(\mathrm{kg} \cdot \mathrm{K}) \\
& \dot{\omega}=\text { species source term in control volume, } \mathrm{kg} /\left(\mathrm{m}^{3} \cdot \mathrm{s}\right) \\
& \dot{w} \quad=\quad \text { species source term in control surface, } \mathrm{kg} /\left(\mathrm{m}^{2} \cdot \mathrm{s}\right) \\
& c^{*}=\text { characteristic velocity, } \mathrm{m} / \mathrm{s} \\
& \mathcal{M}=\text { molar mass, } \mathrm{kg} / \mathrm{kmol} \\
& L \quad=\quad \text { length, } \mathrm{m} \\
& r \quad=\text { radius, } \mathrm{m} \\
& R \quad=\text { port radius, } \mathrm{m} \\
& \mathcal{R}=\text { universal gas constant, } \mathrm{J} /(\mathrm{kmol} \cdot \mathrm{K}) \\
& x=\text { axial coordinate, } \mathrm{m} \\
& \dot{r}=\text { fuel regression rate, } \mathrm{mm} / \mathrm{s} \\
& \dot{m} \quad=\text { mass flow rate, } \mathrm{kg} / \mathrm{s} \\
& y^{+} \quad=\text { dimensionless wall distance for wall-bounded flows } \\
& \varepsilon \quad=\quad \text { surface emissivity } \\
& \epsilon \quad=\text { numerical error } \\
& \mathcal{I}=\text { radiative intensity, } \mathrm{W} /\left(\mathrm{m}^{2} \mathrm{sr}\right)
\end{aligned}
$$

\section{Subscripts}

$$
\begin{aligned}
\mathrm{w} & =\text { wall } \\
\mathrm{T} & =\text { turbulent } \\
\mathrm{cr} & =\text { critical } \\
\mathrm{g} & =\text { gas } \\
i, s & =\text { of species } i, s \\
j & =\text { of reaction } j \\
\mathrm{~s} & =\text { solid } \\
\mathrm{melt} & =\text { melting } \\
\mathrm{conv} & =\text { convection } \\
\text { rad } & =\text { radiation } \\
\text { tot } & =\text { total } \\
\text { th } & =\text { theoretical }
\end{aligned}
$$




\section{Introduction}

YBRID rocket engines (HREs) are propulsion devices usually employing a solid fuel and a gaseous or liquid
oxidizer, stored physically separated from each other. If compared to solid rocket engines, HREs are safer during fabrication, storage, and operations, they allow throttling, shutdown, and restart capabilities, and they present less ambient temperature sensitivity, higher crack robustness of fuel grain, and higher specific impulse. On the other hand, with respect to liquid rocket engines, they are much simpler and cheaper to build, more reliable, and with higher average propellant density. HREs are therefore considered one of the potentially preferred options for specific future generation propulsion systems [1], and have already shown some promising results with the successful flight of the SpaceShipOne [2] and recently with the flight of SpaceShipTwo, and with the ongoing work on the development of the Mars Ascent Vehicle (MAV) [3].

However, one of the most important drawbacks of conventional HREs using classical pyrolyzing fuels such as Hydroxyl-Terminated Poly-Butadiene (HTPB) is the low regression rate of the grain, which entails low thrust levels especially in combination with high-performance oxidizers, such as oxygen, which require lower O/F for maximum efficiency. This shortcoming can be mitigated with the application of different techniques, including multi-port grains, swirl injection, or introduction of fuel additives; however, the most promising one to date is the use of paraffin-based fuels. In fact, contrary to conventional polymeric fuels, which pyrolyze before burning, paraffin-based grains in HREs exhibit a liquid or supercritical fluid layer, depending on the operating pressure, leading to the entrainment of droplets into the gaseous mixture stream [4, 5]. This mechanism allows for a continuous spray of fuel along the port, leading to an additional mass transfer towards the melt layer and the flame front, with most of the fuel vaporization occurring around the droplets. Regression rates up to three to four times higher than the conventional values have been first observed in lab-scale motors and then confirmed in scale-up tests with different oxidizers [6, 7]. High regression rates allow to design high-volumetric-loading single-port combustion chambers, avoiding complex and inefficient multi-port grains. In addition, paraffin-based fuels are non-hazardous, non-toxic, and easy to handle.

The relatively low maturity in the understanding and modeling of the physical phenomena occurring inside paraffinbased HREs, which include fluid-surface interaction, radiation, chemistry, and turbulence, limits their technology readiness level. Modeling strategies available in the literature range from the algebraic models of Marxman's theory [8] and Karabeyoglu's theory for liquefying fuels [4, 5] to Reynolds-averaged Navier-Stokes (RANS) simulations employing different sub-models [9], while direct numerical simulation (DNS) studies are limited to fundamental investigations such as the instability of supercritical liquid films in HRE conditions [10]. RANS simulations tipically rely either on the assignment of a prescribed fuel mass flow rate [11- 15], or employ a parametric gas-surface interaction model, i.e., tuned to match one experimental firing test [16, 17]. All previous numerical studies dealing with paraffin-wax neglect radiation effects, which can be prominent in HRE combustion chambers [18--20], and, in addition, neglect its thermal cracking reaction by directly injecting ethylene from the fuel grain surface to the flow field. One open challenge is the 
ability to predict the internal ballistic of paraffin-based HREs without compromising the simplicity and inclusiveness of the model strategy, and without tuning to experimental data.

In this work, we attempt to provide such a predictive model by building upon previously presented preliminary results [21-23], and validate it through a rebuilding of the experimental data taken from a recent firing test of one paraffin-based HRE [15]. The computational approach is able to compute the correct flowfield, capture the fluid-surface interaction without losing predictive capabilities, and account for the radiative heat transfer. A particular hypothesis employed is that, in supercritical conditions, the turbulent diffusion and convection of liquefied paraffin-wax occurs similarly to that of the other species constituting the mixture and, as a consequence, that the entrainment is part of the turbulent mixing process. The resulting single-phase RANS approach should therefore be valid at supercritical turbulent conditions, also considering that recent experimental visualizations of paraffin-based combustion have shown that the entrainment phenomenon typical of subcritical combustion is modified at elevated pressures [24, 25].

The manuscript is organized as follows. The mathematical and computational model are presented first including the chemical kinetic model for the gaseous phase, the approach followed for fluid-surface interaction, and the radiative wall heat transfer model (Section III). Then, the geometrical setup for all simulations is presented together with the main features of the ten test cases that will be discussed (Section [III). In Section IV] the results from the numerical simulations are first evaluated through a grid sensitivity analysis, then they are discussed, and they are finally compared with experimental data. Eventually, a summary of the main conclusions is reported in Section $\square$

\section{Computational Model}

\section{A. Fluid Dynamics Model}

Numerical computations are performed by solving the RANS equations for three-dimensional, turbulent, compressible, multi-component, and reacting flows [26]:

$$
\begin{aligned}
& \frac{\partial\left(\rho y_{i}\right)}{\partial t}+\nabla \cdot\left(\rho \mathbf{v} y_{i}\right)=-\nabla \cdot \mathbf{j}_{i}+\dot{\omega}_{i} \quad(i=1, \ldots, N) \\
& \frac{\partial(\rho \mathbf{v})}{\partial t}+\nabla \cdot(\rho \mathbf{v v})=\nabla \cdot \mathbf{S} \\
& \frac{\partial(\rho E)}{\partial t}+\nabla \cdot(\rho E \mathbf{v})=\nabla \cdot(\mathbf{v} \cdot \mathbf{S})-\nabla \cdot \mathbf{q}
\end{aligned}
$$


where $E$ is total energy per unit mass, and the mass diffusion flux vector of the $i$-th species, the stress tensor, and the heat flux vector are, respectively,

$$
\begin{aligned}
\mathbf{j}_{i} & =-\left(\frac{\mu}{\mathrm{Sc}}+\frac{\mu_{\mathrm{T}}}{\mathrm{Sc}_{\mathrm{T}}}\right) \nabla y_{i} \\
\mathbf{S} & =-p \mathbf{I}-\left(\mu+\mu_{\mathrm{T}}\right)\left\{\frac{2}{3}(\nabla \cdot \mathbf{v}) \mathbf{I}+\left[\nabla \mathbf{v}+(\nabla \mathbf{v})^{\mathrm{T}}\right]\right\} \\
\mathbf{q} & =-\left(k+\frac{\mu_{\mathrm{T}}}{\operatorname{Pr}_{\mathrm{T}}} \sum_{i=1}^{N} y_{i} c_{p, i}\right) \nabla T+\sum_{i=1}^{N}\left(h_{i}+\Delta h_{\mathrm{f}, i}^{T_{\mathrm{ref}}}\right) \mathbf{j}_{i}
\end{aligned}
$$

where $\mathbf{I}$ is the unit tensor, Pr the Prandtl number, and Sc the Schmidt number. Thermodynamic closure is achieved with the thermally perfect ideal gas equation of state.

The sensible enthalpy $h_{i}$ and the corresponding specific isobaric heat $c_{p, i}$ for the $i$-th species (apart from paraffin, discussed separately in section [I.B] are expressed as a function of temperature according to the seventh-order polynomials reported in the chemical equilibrium with applications (CEA) database [27], from which the values of the standard heat of formation at the reference temperature $\Delta h_{\mathrm{f}, i}^{T_{\mathrm{ref}}}$ are also taken. The mixture molecular transport properties $\mu$ and $k$ are obtained according to Wilke's rule [26] from those of the individual species, which are obtained from the fourth-order polynomials of temperature reported in [27] (apart from paraffin), while species molecular diffusivity is considered to be the same for all species through a constant Schmidt number, Sc $=0.7$. In fact, molecular diffusion phenomena are not expected to play a significant role in the heat-transfer-controlled problem analyzed in this study. The turbulent viscosity, $\mu_{\mathrm{T}}$, is evaluated through the integration of an additional convection/diffusion equation, according to the Spalart-Allmaras one-equation model [28], whose standard constants are used for model closure. Turbulent diffusivity and conductivity are evaluated on the basis of $\mu_{\mathrm{T}}$ through constant turbulent Schmidt and Prandtl numbers, $\mathrm{Sc}_{\mathrm{T}}=0.7$ and $\operatorname{Pr}_{\mathrm{T}}=0.9$, respectively.

The chemical source terms $\dot{\omega}_{i}$ in Eq. (1) are obtained as the contribution of each of the $N_{\mathrm{r}}$ reactions,

$$
\dot{\omega}_{i}=\mathcal{M}_{i} \sum_{j=1}^{N_{\mathrm{r}}}\left(v_{i, j}^{\mathrm{P}}-v_{i, j}^{\mathrm{R}}\right)\left[k_{\mathrm{f}, j} \prod_{s=1}^{N}\left(\frac{\rho_{s}}{\mathcal{M}_{s}}\right)^{v_{s, j}^{\mathrm{R}}}-k_{\mathrm{b}, j} \prod_{s=1}^{N}\left(\frac{\rho_{s}}{\mathcal{M}_{s}}\right)^{v_{s, j}^{\mathrm{P}}}\right]
$$

where the generic reaction among species $B_{i}$ is expressed with the stoichiometric coefficients of reactants $v_{i, j}^{\mathrm{R}}$ and products $v_{i, j}^{\mathrm{P}}$ as

$$
\sum_{i=1}^{N} v_{i, j}^{\mathrm{R}} B_{i} \rightleftarrows \sum_{i=1}^{N} v_{i, j}^{\mathrm{P}} B_{i} \quad\left(j=1, \ldots, N_{\mathrm{r}}\right)
$$

The forward $k_{\mathrm{f}, j}$ reaction rate is expressed as

$$
k_{\mathrm{f}, j}=A_{j} T^{n_{j}} \exp \left(-\frac{E_{\mathrm{a}, j}}{\mathcal{R} T}\right)
$$


Table 1 Global kinetic mechanism for paraffin-wax/oxygen combustion (with units of kilomoles, meters, and seconds).

\begin{tabular}{llcccc}
\hline \hline$j$ & Reaction & $A_{j}$ & $n_{j}$ & $E_{\mathrm{a}, j} / \mathcal{R}(\mathrm{K})$ & \multicolumn{2}{c}{ Reference } \\
\hline 1 & $\mathrm{C}_{32} \mathrm{H}_{66} \longrightarrow 16 \mathrm{C}_{2} \mathrm{H}_{4}+\mathrm{H}_{2}$ & $3.09 \cdot 10^{13}$ & 0 & 29791 & [32] \\
2 & $\frac{1}{2} \mathrm{C}_{2} \mathrm{H}_{4}+\frac{5}{4} \mathrm{O}_{2} \longrightarrow 2 \mathrm{CO}+2 \mathrm{H}_{2}-\frac{1}{2} \mathrm{C}_{2} \mathrm{H}_{4}+\frac{1}{4} \mathrm{O}_{2}$ & $9.11 \cdot 10^{13}$ & 0 & 15702 & [30] \\
3 & $\mathrm{C}_{2} \mathrm{H}_{4}+\mathrm{H}_{2} \mathrm{O} \longrightarrow 2 \mathrm{CO}+4 \mathrm{H}_{2}-\mathrm{H}_{2} \mathrm{O}$ & $3.48 \cdot 10^{11}$ & 0 & 15400 & {$[30]$} \\
4 & $\mathrm{CO}+\mathrm{H}_{2} \mathrm{O} \rightleftarrows \mathrm{CO}_{2}+\mathrm{H}_{2}$ & $2.90 \cdot 10^{12}$ & 0 & 9613 & {$[30]$} \\
5 & $\mathrm{H}_{2}+\frac{1}{2} \mathrm{O}_{2} \rightleftarrows \mathrm{H}_{2} \mathrm{O}$ & $2.80 \cdot 10^{18}$ & -1 & 21691 & {$[30]$} \\
6 & $\mathrm{O}_{2} \rightleftarrows 2 \mathrm{O}$ & $1.50 \cdot 10^{9}$ & 0 & 56870 & {$[30]$} \\
7 & $\mathrm{H}_{2} \mathrm{O} \rightleftarrows \mathrm{OH}+\mathrm{H}$ & $2.30 \cdot 10^{22}$ & -3 & 60393 & {$[30]$} \\
\hline \hline
\end{tabular}

where $A_{j}$ is the pre-exponential factor, $n_{j}$ the temperature exponent, and $E_{\mathrm{a}, j}$ the molar activation energy. The backward $k_{\mathrm{b}, j}$ reaction rate, which is not null only for reversible reactions, is expressed as

$$
k_{\mathrm{b}, j}=k_{\mathrm{f}, j} / K_{j}
$$

where $K_{j}$ is the equilibrium constant of the $j$-th reaction evaluated from thermodynamic data taken from [29].

In the present study, a global kinetic mechanism used for butadiene combustion [30] is adapted to the combustion of ethylene, one of the main products of paraffin-wax thermal cracking [31, 32], because the authors have not found any similar global kinetic mechanism for ethylene in the literature. The global reaction mechanism for gaseous oxygen and paraffin-wax combustion ultimately involves seven reactions and ten species (see Table 1 ). The reaction rate constant of paraffin thermal cracking presented in Ref. [32] is valid for liquid $\mathrm{C}_{16} \mathrm{H}_{34}$, but it is assumed here to be valid also for that of $\mathrm{C}_{32} \mathrm{H}_{66}$ because of the lack of data in the literature.

The RANS equations are numerically solved up to the wall with an in-house Computational Fluid Dynamics (CFD) solver that has been validated against experimental data in different operating conditions [33-39]. The solver adopts a standard cell-centered finite volume Godunov-type formulation with second-order accuracy in space on multi-block structured meshes, and employs a Roe approximate Riemann solver [40]. Time integration is performed with the Strang operator-splitting technique [41]: convective and diffusive terms are integrated by a second-order Runge-Kutta scheme, whereas for the chemical source terms an implicit integrator for stiff ordinary differential equations is used [42].

\section{B. Paraffin Modeling and Fluid-Surface Interaction}

\section{Thermodynamic and transport properties of paraffin-wax}

The paraffin taken into account in the present manuscript is $\mathrm{C}_{32} \mathrm{H}_{66}$ (see Table 2 for its properties), according to the test case we want to simulate. The critical pressure of the selected paraffin-wax, equal to 6.5 bar [44], is typically below the pressure achieved in HRE combustion chambers. Accordingly, paraffin-wax is assumed here to be in the 
Table 2 Paraffin-wax $\mathrm{C}_{32} \mathrm{H}_{66}$ properties.

\begin{tabular}{ccccccc}
\hline \hline $\mathcal{M}$ & $T_{\text {melt }}[43]$ & $\Delta h_{\text {melt }}[43]$ & $\rho_{\mathrm{s}}[17]$ & $c_{\mathrm{s}}[43]$ & $T_{\mathrm{s}, \text { in }}$ & $\Delta h_{\mathrm{f}}^{T_{\text {ref }}}[43]$ \\
\hline $450.87 \mathrm{~kg} / \mathrm{kmol}$ & $343 \mathrm{~K}$ & $169.83 \mathrm{~kJ} / \mathrm{kg}$ & $920 \mathrm{~kg} / \mathrm{m}^{3}$ & $1946.03 \mathrm{~J} /(\mathrm{kg} \cdot \mathrm{K})$ & $298.15 \mathrm{~K}$ & $-967.8 \mathrm{~kJ} / \mathrm{mol}$ \\
\hline \hline
\end{tabular}

supercritical pressure regime, where no surface tension can be defined and no boundary for droplets exists [45, 46]. In such conditions it is reasonable to assume that the turbulent diffusion and convection of the supercritical species occurs similarly to that of the other species constituting the mixture and, as a consequence, that the entrainment is part of the turbulent mixing process. Therefore, a classical single-phase RANS approach can still be employed for predictive purposes.
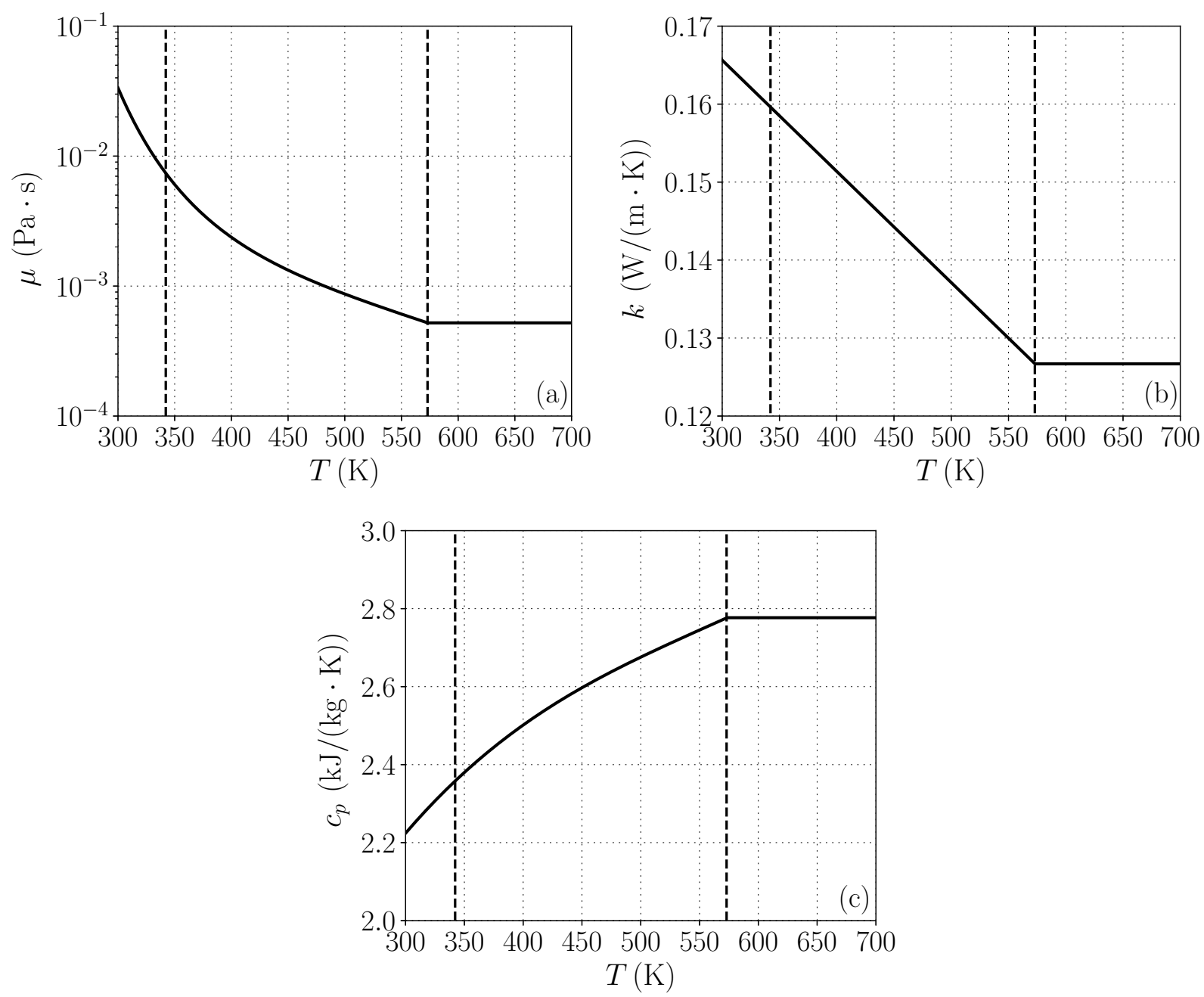

Fig. 1 Thermodynamic and transport properties of paraffin-wax $\mathrm{C}_{32} \mathrm{H}_{66}$ obtained with the model of [44, 47]: dynamic viscosity (a), thermal conductivity (b), and specific isobaric thermal capacity (c). The vertical dashed lines are drawn at $T=T_{\text {melt }}$ and $T=573 \mathrm{~K}$ (limit for the validity of the model), respectively. 
The melted species is described through a simplified dense fluid approach [21, 23], with thermodynamic and transport properties taken from the liquid-like paraffin-wax model described in [47]. The specific isobaric thermal capacity, specific enthalpy, and specific entropy $s$ are a function of temperature only and can be computed with:

$$
\begin{gathered}
\frac{c_{p}}{R}=a_{1}+a_{2} T+a_{3} T^{2}+a_{4} T^{3} \\
\frac{h}{R T}=a_{1}+a_{2} \frac{T}{2}+a_{3} \frac{T^{2}}{3}+a_{4} \frac{T^{3}}{4}+\frac{b_{1}}{T} \\
\frac{s}{R}=a_{1} \ln T+a_{2} T+a_{3} \frac{T^{2}}{2}+a_{4} \frac{T^{3}}{3}+b_{2}
\end{gathered}
$$

where $R=\mathcal{R} / \mathcal{M}$, and the coefficients are $a_{1}=4.98, a_{2}=6.36 \cdot 10^{-1} \mathrm{~K}^{-1}, a_{3}=-1.02 \cdot 10^{-3} \mathrm{~K}^{-2}, a_{4}=6.15 \cdot 10^{-7} \mathrm{~K}^{-3}$, $b_{1}=-1.15 \cdot 10^{5} \mathrm{~K}, b_{2}=-5.79 \cdot 10^{2}$. The model of Marano and Holder [47] allows to obtain the dynamic viscosity, in $\mathrm{Pa} \cdot \mathrm{s}$, as

$$
\mu_{\mathrm{C}_{32} \mathrm{H}_{66}}=0.001 \exp \left(A_{\mu}+B_{\mu} / T+C_{\mu} \ln T+D_{\mu} T^{2}+E_{\mu} / T^{2}\right)
$$

where $A_{\mu}=1.05 \cdot 10^{2}, B_{\mu}=-1.42 \cdot 10^{4} \mathrm{~K}, C_{\mu}=-1.35 \cdot 10^{1}, D_{\mu}=-3.16 \cdot 10^{-6} \mathrm{~K}^{-2}, E_{\mu}=2.13 \cdot 10^{6} \mathrm{~K}^{2}$, and the thermal conductivity, in $\mathrm{W} /(\mathrm{m} \cdot \mathrm{K})$, as

$$
k_{\mathrm{C}_{32} \mathrm{H}_{66}}=A_{k}+B_{k} T
$$

where $A_{k}=2.08 \cdot 10^{-1}$ and $B_{k}=-1.43 \cdot 10^{-4} \mathrm{~K}^{-1}$. For temperatures exceeding $573 \mathrm{~K}$ (limit value for the validity of the model), constant properties are assumed (see Fig. 11). Transport properties of paraffin are mostly relevant to the prediction of the internal ballistics in the viscous sublayer next to the fuel grain, whose composition is dominated by liquefied paraffin at temperatures lower than $573 \mathrm{~K}$. The assumptions employed in this work can be considered fairly reasonable when the fluid actually tends to behave like a pressurized liquid, that is at temperatures below $860 \mathrm{~K}$, which is the critical temperature of paraffin-wax [44], whereas gaseous-like properties should be considered at higher temperatures. However, this approximation does not hinder the correct prediction of the internal ballistics because, as a consequence of thermal cracking, most of the liquefied paraffin in the flow field is at temperatures lower than the critical temperature.

\section{Fluid-surface interaction}

An adequate boundary condition, based on mass and energy balances for a control volume fixed to the fluid-surface interface, has to be specified in order to describe the physics of surface phenomena.

The mass balance is given by

$$
\rho D \frac{\partial y_{i}}{\partial \eta}+\dot{w}_{i}=\dot{r} \rho_{\mathrm{s}} y_{i} \quad i=1, \ldots, N
$$


where $\eta$ is the coordinate normal to the grain (from solid to fluid) and $D$ is the diffusion coefficient, and expresses for each species the balance between the mass entering the control volume due to diffusion and melting, and the mass leaving the volume by convection.

The energy balance is given by

$$
q_{\mathrm{w}, \mathrm{conv}}+q_{\mathrm{w}, \mathrm{rad}}+\sum_{i=1}^{N} h_{i} \rho D \frac{\partial y_{i}}{\partial \eta}+\dot{r} \rho_{\mathrm{s}} h_{\mathrm{s}}=\dot{r} \rho_{\mathrm{s}} h_{\mathrm{w}}+\dot{r} \rho_{\mathrm{s}} c_{\mathrm{s}}\left(T_{\mathrm{melt}}-T_{\mathrm{s}, \text { in }}\right)
$$

where $q_{\mathrm{w}, \mathrm{conv}}=k \partial T / \partial \eta$ and $T_{\mathrm{s} \text {,in }}$ is the initial temperature of the fuel grain, and expresses the balance between the energy entering the control volume because of convection, radiation, diffusion, and regression, and the energy leaving the volume because of convection and conduction. The wall temperature is considered equal to the paraffin-wax melting temperature. The expression for the conductive heat flux follows from the steady-state assumption for temperature in a reference system moving with the receding melting boundary [48]. This is a reasonable approximation when the thermal lag in the solid is sufficiently small, as actually occurs in the simulated conditions because of the moderately high regression rates and the low thermal conductivity of the fuel grain. In fact, by considering $k=0.325 \mathrm{~W} /(\mathrm{m} \cdot \mathrm{K})$ [49], hence a thermal diffusivity of $\alpha=1.82 \cdot 10^{-7} \mathrm{~m}^{2} / \mathrm{s}$, the thermal penetration depths expected for regression rates of $\dot{r}=2 \mathrm{~mm} / \mathrm{s}$ are of the order of $\approx 0.3 \mathrm{~mm}(3 \alpha / \dot{r})$, much smaller than the fuel grain thickness, and the times to reach steady state conduction of $\approx 0.1 s\left(2 \alpha / \dot{r}^{2}\right)$, much less than the operational times [50, 51].

Substituting Eq. (12) into Eq. (13), the energy balance can be recast as

$$
q_{\mathrm{w}, \mathrm{conv}}+q_{\mathrm{w}, \mathrm{rad}}=\dot{r} \rho_{\mathrm{s}}\left[\Delta h_{\mathrm{melt}}+c_{\mathrm{s}}\left(T_{\mathrm{melt}}-T_{\mathrm{s}, \text { in }}\right)\right]
$$

where

$$
\dot{r} \rho_{\mathrm{s}} \Delta h_{\mathrm{melt}}=\dot{r} \rho_{\mathrm{s}}\left(\sum_{i=1}^{N} \frac{\dot{w}_{i}}{\dot{r} \rho_{\mathrm{s}}} h_{i}-h_{\mathrm{s}}\right)
$$

is the chemical heat flux. Equation (14) emphasizes that the energy balance is established between the heat transferred to the grain via convection and radiation and the heat absorbed because of phase change and thermal conduction. Equation (14) allows evaluating the fuel mass flux injected into the combustion chamber, $\dot{r} \rho_{\mathrm{s}}$, and the corresponding regression rate is computed at the post-processing stage. Since the mass flux at wall is equal to the liquefied paraffin-wax source term, $\dot{w}_{\mathrm{C}_{32} \mathrm{H}_{66}}$, the mixture composition at wall can be computed solving Eq. (12) for each species.

The wall boundary condition based on the described fluid-surface interaction model has been previously validated for ablating surfaces in re-entry flows [52] and solid-rocket nozzles [53], while it has been already extended to hybrid rocket applications for nozzle erosion [54] and pyrolysing fuel grains regression problems [39, 55, 56]. 


\section{Thermal radiation model}

Thermal radiation modeling is essential for the determination of the radiative wall heat flux needed by the fluid-surface interaction balance equation [Eq. [14]]. The modeling strategy, which has been already used in previous studies of liquid rockets [22, 57], hybrid rockets with polymeric fuels [56], and paraffin-based fuels [58] relies on the main assumptions of gray/diffuse wall and of gray/non-scattering medium, which are justified below. The global treatment of spectral features, leading to the assumption of gray wall, is deemed to be quite accurate given its fair independence from wavelength over the spectrum. The constraint on computer time restricts the choice to the gray assumption also for the gas, enabling to compute a single radiative intensity. The high roughness level expected for the receding surface, moreover, justifies the diffuse wall hypothesis, since reflection from rough surfaces approximates the diffuse reflection regime [59]. The absence of solid particles suspended in the gas phase, finally, is consistent with the assumption of a non-scattering behavior of the medium, and the effect of soot radiation is not accounted for in the present study. It is also assumed that radiation does not affect the flow field significantly [60], because of the relative small weight of the wall heat transfer, and in particular of the radiative contribution, as compared to the whole thermal power generated within the thrust chamber. This assumption allows to evaluate the radiative heat flux only at the boundaries, and to neglect its contribution into the energy conservation equation.

The incident radiative heat flux reaching a specific wall location is defined by the integral of the wall radiative intensity over the hemispherical solid angle facing the incoming radiation,

$$
q_{g, \mathrm{rad}}=\int_{4 \pi} \mathcal{I}_{\mathrm{w}} \sin \theta d \Omega=\int_{0}^{2 \pi} \int_{0}^{\frac{\pi}{2}} \mathcal{I}_{\mathrm{w}} \sin \theta \cos \theta d \theta d \psi
$$

where $\Omega$ is the solid angle, and $\theta$ and $\psi$ are the line-of-sight elevation and azimuth angle, respectively. The radiative intensity at the wall from a generic line-of-sight can be computed by integrating the radiative transfer equation (RTE) along the whole radiation path length. The RTE expresses the balance of radiative intensity along a generic direction, including contributions due to absorption/emission and, potentially, in/out-scattering. Under the assumption of gray/non-scattering medium, the RTE reduces to the form

$$
\frac{d \mathcal{I}}{d s}=j^{e}-\kappa \mathcal{I}
$$

where $j^{e}$ is the power per unit volume emitted by the gas, $\kappa$ is the absorption coefficient, and $s$ is the abscissa along a lineof-sight. The term $j^{e}$ can be expressed as proportional to the black-body radiative intensity through a proportionality constant for emission equal to the absorption coefficient, i.e. $j^{e}=\kappa \sigma T^{4} / \pi$, where $\sigma$ is the Stefan-Boltzmann constant. 
The RTE formal solution can then be obtained as

$$
\mathcal{I}_{\mathrm{w}}=\mathcal{I}_{\mathrm{w}, 0} \exp \left(-\int_{s_{\mathrm{w}, 0}}^{s_{\mathrm{w}}} \kappa d s\right)+\int_{s_{\mathrm{w}, 0}}^{s_{\mathrm{w}}} j^{e} \exp \left(-\int_{s}^{s_{\mathrm{w}}} \kappa d s^{\prime}\right) d s
$$

where the line-of-sight originates from another wall point (indicated with the subscript 0 ).

Equations $(16)$ and $(18)$ require the knowledge of radiative intensity at the line-of-sight origin $\mathcal{I}_{\mathrm{w}, 0}$ and of the absorption coefficient of the gas mixture $\kappa$ to be solved. The former, under the assumption of gray/diffuse wall, can be simply evaluated taking into account both the emitted and reflected radiative intensity,

$$
\pi \mathcal{I}_{\mathrm{w}, 0}=\varepsilon_{\mathrm{w}, 0} \sigma T_{\mathrm{w}, 0}^{4}+\left(1-\varepsilon_{\mathrm{w}, 0}\right) q_{\mathrm{g}, \mathrm{rad}, 0}
$$

The latter is derived by means of a global model, typically used for high-temperature combustion mixtures under vibrational equilibrium conditions,

$$
\kappa=p \sum_{i=1}^{N} X_{i} \kappa_{p, i}
$$

indicating that the absorption of radiative energy is proportional to the pressure and to the absorption coefficients of the participating species weighted with their molar fraction $X_{i}$. Note that $\kappa_{p, i}=0$ except for $\mathrm{H}_{2} \mathrm{O}, \mathrm{CO}_{2}$, and $\mathrm{CO}$, which are the most relevant in the process of thermal radiation exchange [61, 62]. Radiation from hydroxyls, widely exploited for combustion diagnostics, is not considered relevant under the energetic standpoint for rocket chamber conditions, despite the non-negligible $\mathrm{OH}$ concentrations [63, 64]. The absorption coefficients averaged over the whole spectrum, i.e. the Planck mean absorption coefficients, are obtained from the model of [63]. This model, not explicitly accounting for high-pressure effects, does admittedly leave some uncertainty, which is however deemed of a weight comparable to those implied by other aspects of the model.

In the present work, the RTE is integrated with the discrete transfer method (DTM) using an in-house software for generic axisymmetric gray/diffuse boundaries and inhomogeneous gray/non-scattering media. The software was suitably developed and validated in [22, 57]. For DTM simulations, a discretization consisting in 256 rays for each calculation point and a step of $1 \mathrm{~mm}$ along each ray have been used after performing convergence analyses for both parameters. Moreover, a wall emissivity equal to 0.91 has been assumed for paraffin-wax by using the emissivity model proposed in [65]. The outlet section is modeled as an open surface, neglecting its contribution of emission and reflection.

Once the incident radiative heat flux is obtained with Eq. [16), the net radiative wall heat flux can be computed with

$$
q_{\mathrm{w}, \mathrm{rad}}=\varepsilon_{\mathrm{w}}\left(q_{g, \mathrm{rad}}-\sigma T_{\mathrm{w}}^{4}\right)
$$

where the first term on the right hand side is the absorbed heat flux, according to Kirkhoff's law, and the last is the 
Table 3 Average port radius and oxygen mass flow rate (see Fig. 2) of the test cases considered in this study, taken from the firing tests of [15].

\begin{tabular}{ccccccccccc}
\hline \hline Test & 1 & 3 & 4 & 8 & 9 & 10 & 11 & 12 & $1 \mathrm{~W}$ & $2 \mathrm{~W}$ \\
\hline$R(\mathrm{~mm})$ & 10.25 & 11.80 & 12.65 & 14.25 & 14.50 & 14.50 & 14.95 & 14.00 & 13.55 & 13.55 \\
$\dot{m}_{\mathrm{ox}}(\mathrm{g} / \mathrm{s})$ & 16.0 & 29.0 & 39.0 & 44.0 & 50.2 & 55.5 & 60.0 & 59.5 & 42.0 & 60.5 \\
\hline \hline
\end{tabular}

emitted heat flux according to the corresponding total black-body emissive power $\sigma T_{\mathrm{w}}^{4}$. Equation 21 shows that $q_{\mathrm{w}, \text { rad }}$ depends on the gas properties (see Eq. $(16)$ ), on the wall temperature, and on the wall properties $\left(\varepsilon_{\mathrm{w}}\right.$ and $\mathcal{I}_{\mathrm{w}, 0}$, see Eq. (19)).

The CFD and DTM codes are coupled with the procedure outlined in the following. First, a tentative profile of the radiative wall heat flux is guessed in order to generate the first CFD solution. The flowfield and wall temperature are then given as inputs to the DTM code, which in turn provides the corresponding radiative wall heat flux. The latter is then used for the enforcement of the boundary condition on the grain surface in the next step of the CFD code, up to convergence to the final solution in 2-3 iterations.

\section{Computational Setup}

Aiming to validate the predictive capabilities of the present approach, ten experimental firing tests carried out at University of Naples "Federico II" [15] are considered in the present study (see Table 3).

The present RANS simulations have all a common axisymmetric geometrical setup (see Fig. 22). More specifically, the cylindrical combustion chamber is made of a pre-chamber $\left(0<x<x_{0}\right)$, the chamber with the paraffin grain $\left(x_{0}<x<x_{1}\right)$, a post-chamber $\left(x_{1}<x<x_{2}\right)$, and finally a converging-diverging nozzle $\left(x_{2}<x<L\right)$. The latter is composed by conical converging and diverging sections connected by circular arcs with each other and with the cylindrical post-chamber. On the left hand side of the setup, a subsonic inflow boundary condition imposing mass flow rate and static temperature simulates an oxygen injector, with the injector plate modeled as an adiabatic wall. On the top side, adiabatic walls are imposed outside of the paraffin grain section, where the boundary condition based on mass and energy balance, described in Section II.B.2, is imposed. Finally, a symmetry condition is applied at the centerline because of the axisymmetric flow assumption, and a supersonic outflow is assumed at the outlet section. For all cases, $L=336.17 \mathrm{~mm}, x_{0}=25 \mathrm{~mm}, x_{1}=245 \mathrm{~mm}, x_{2}=303 \mathrm{~mm}, r_{\text {inj }}=3 \mathrm{~mm}, r_{\mathrm{t}}=5.3 \mathrm{~mm}$, and $r_{\mathrm{e}}=8.3$ $\mathrm{mm}$, while the average port radius $\mathrm{R}$ varies for each test case (see Table 3). A single simulation at the average port diameter is performed for each test case. The actual radius of pre-chamber and post-chamber are different from that of the cylindrical grain port. However, based on foregoing results [39], they have been shown to affect only slightly the regression rate, and are therefore assumed with the same radius of the cylindrical port in the present study. This 


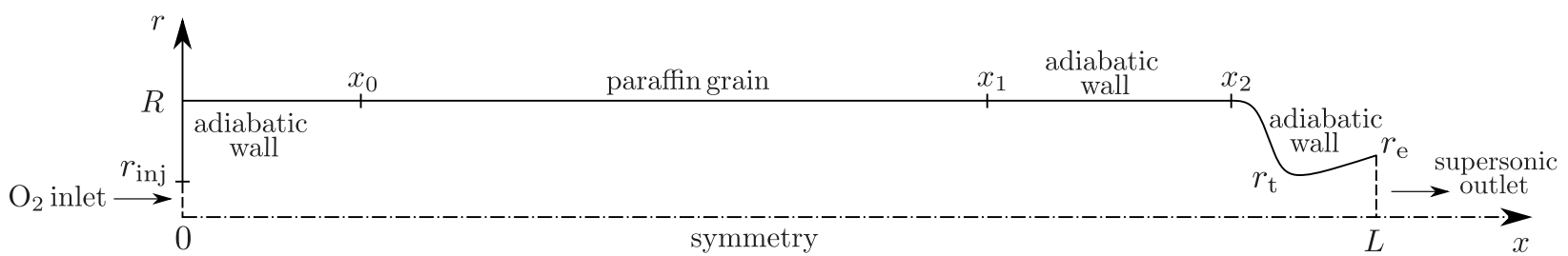

Fig. 2 Computational setup used for the axisymmetric numerical simulations.

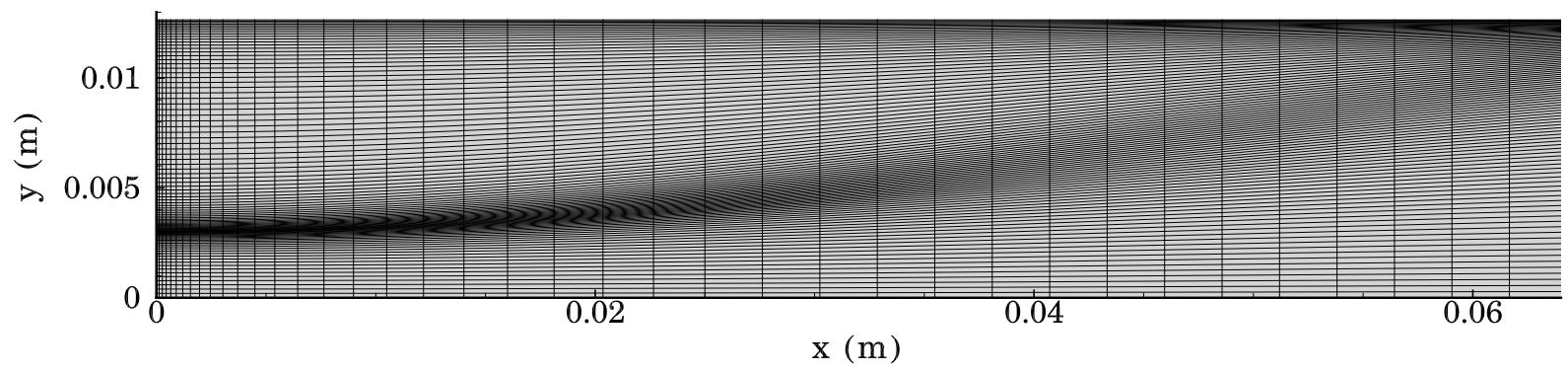

Fig. 3 Enlargement of the injector region showing the 160x100 mesh used for the numerical simulations of test 4 (see Table 3).

simplification may have an effect on the $c^{*}$ efficiency which will be discussed later.

The reference mesh employed in this work is divided into 160 grid points in the axial direction, and 100 grid points in the radial direction. A proper grid clustering is introduced in the injection (see Fig. 3), near-wall, and throat regions to sufficiently resolve the mixing layer, the boundary layer, and the transition through sonic conditions, respectively.

\section{Results and Discussion}

The results from the Reynolds-averaged Navier-Stokes simulations show several peculiarities of hybrid rockets burning paraffin-wax and gaseous oxygen. We start with a grid sensitivity analysis, followed by a description of the flow field including a discussion on the effect of radiation, and finally we conclude with the rebuilding of the time-averaged experimental regression rate, chamber pressure, and combustion efficiency.

\section{A. Grid sensitivity analysis}

Three different grids have been considered for the grid sensitivity analysis. Test case 4 (see Table 4) has been selected for this purpose. A coarse grid, which is made by $80 \times 50$ cells, is obtained by halving the number of cells in both axial and radial directions with respect to the reference grid. A fine grid composed of 320x200 cells is obtained instead by doubling the number of cells in both directions with respect to the reference grid. The characteristic spatial discretization $\Delta x$ is therefore always halved by refining the mesh.

A fairly accurate representation of the flow field can be obtained with the coarse grid, i.e. with just 4000 control volumes. In fact, it was observed that the fuel regression rate is weakly sensitive to wall resolution if $y^{+}<3$ (see 
Table 4 Meshes considered for the grid refinement study.

\begin{tabular}{cccc}
\hline \hline & coarse & medium & fine \\
\hline Mesh & $80 \times 50$ & $160 \times 100$ & $320 \times 200$ \\
Number of control volumes & 4000 & 16000 & 64000 \\
Maximum cell height on grain (test 4) & $25.25 \mu \mathrm{m}$ & $12.34 \mu \mathrm{m}$ & $6.10 \mu \mathrm{m}$ \\
Maximum $y^{+}$on grain (test 4) & 2.94 & 1.42 & 0.69 \\
\hline \hline
\end{tabular}

Fig. 4a), but only with a mesh of 160x100 cells a grid-independent solution is obtained (Fig. 4 p). The boundary layer tends to become thinner with increasing distance from the reattachment point, which corresponds to the minimum $y+$ in Fig. 4h, yielding a gradual increase of $y^{+}$with $x$. The profile of regression rate obtained with the fine mesh varies with respect to the medium discretization level only slightly at the beginning of the grain, and its integral average varies of $2.5 \%$ between the coarse and fine mesh, and of $0.03 \%$ between the medium and fine mesh. Monotonic convergence of chamber pressure is observed, with variations between the medium and fine mesh of less than $1 \%$ (Fig. 4f). The flow field does not show significant changes with increasing mesh resolution from the medium to the fine level, as the radial profiles shown in Fig. $4 \mathrm{~d}$ confirm. Note that at mid grain the three different resolutions provide superimposed temperature profiles. Therefore, the 160x100 mesh is considered acceptable for all the test cases in Table 3

From the grid sensitivity analysis it is found that the numerical error reduces roughly following the spatial order of accuracy of the scheme. The error can therefore be reasonably evaluated by calculating the asymptotic value according to the Richardson-extrapolation procedure [66, 67] with $n=2$, which for pressure errors reads

$$
p_{\mathrm{RE}}=p_{\text {fine }}+\frac{p_{\text {fine }}-p_{\text {medium }}}{n^{2}-1} \quad ; \quad \epsilon=\left|p-p_{\mathrm{RE}}\right| / p_{\mathrm{RE}}
$$

The numerical error on pressure and on regression rate computed with this procedure is shown in Fig. 5

\section{B. Description of the flow field}

A wide diffusion flame is observed throughout the engine for test 4 (Fig. 6a). The flame is anchored to the injector's edge, due to the recirculation region entailed by the axial injection of oxygen (see inset in Fig. 6a). A large fraction of prevalently cold and unburnt paraffin fuel circulates in the pre-chamber region (Fig. 6p), consistent with post-firing inspections of the injector head [15]. The recirculation region is not confined only in the pre-chamber region, but, in fact, more than half of the vortex develops on the paraffin grain. This causes the melted paraffin-wax injected from the solid grain, and generally present mainly in a narrow layer close to the grain surface, to move upstream towards the injector. After the reattachment point, appearing on the fuel grain, the liquid paraffin-wax accumulates with increasing axial distance, covering the post-chamber and partially also the nozzle walls. The flame widens and gets closer to the grain, 


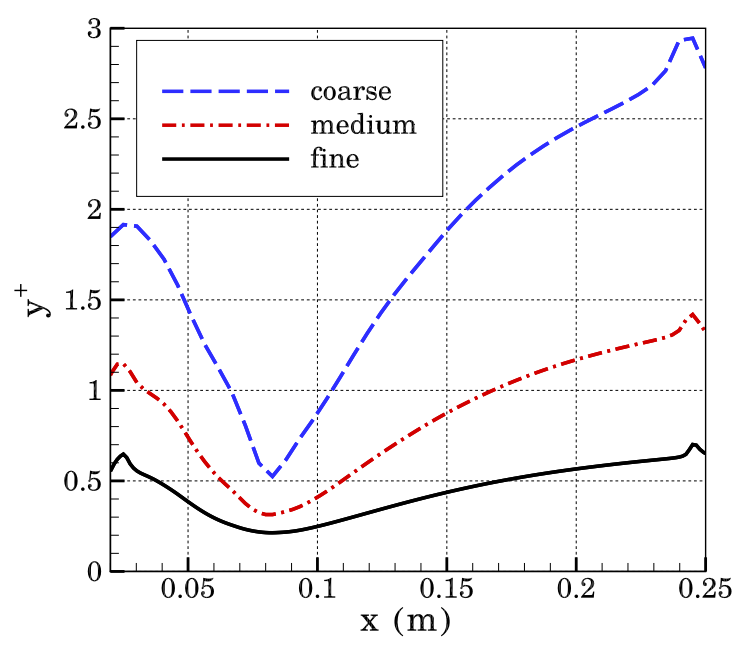

(a) Dimensionless wall distance for wall-bounded flows $\left(y^{+}\right)$.

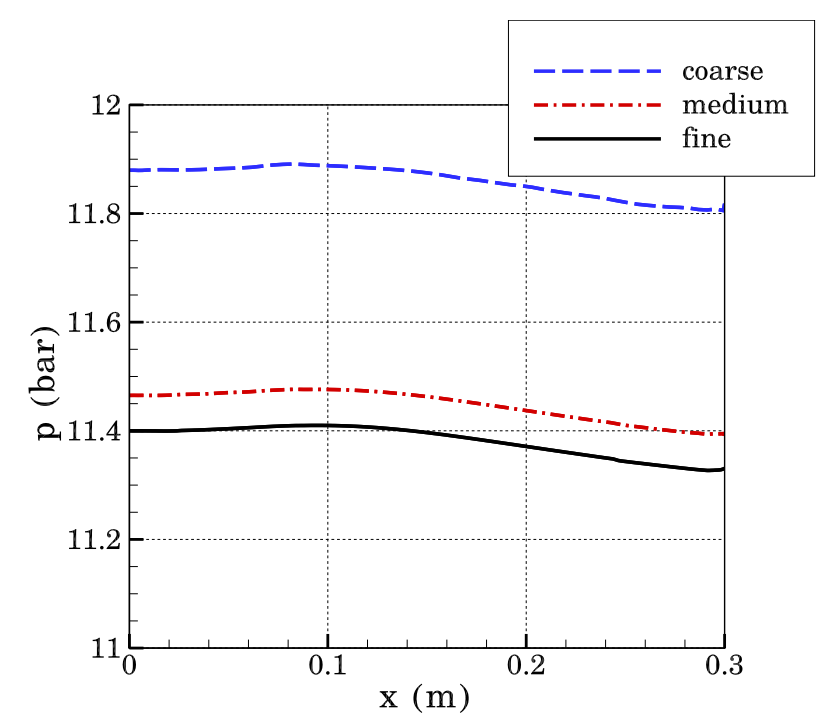

(c) Wall pressure.

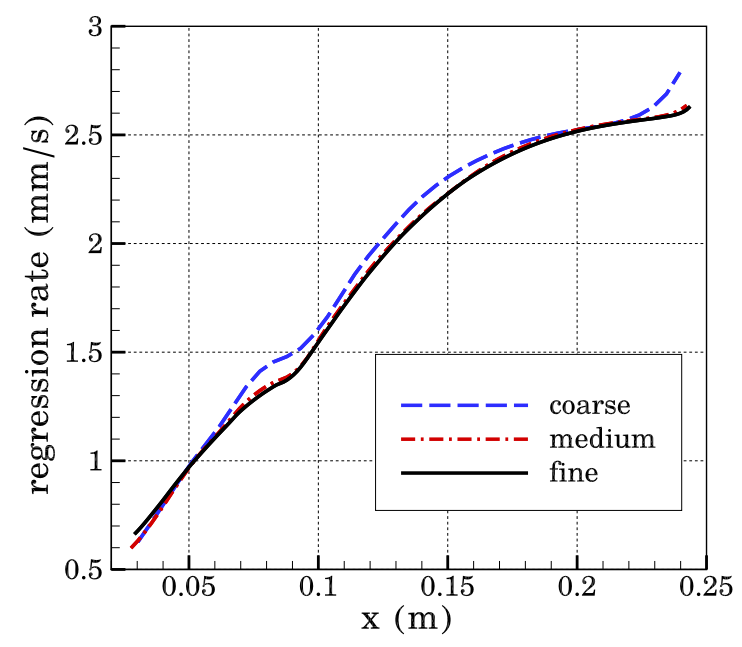

(b) Regression rate.

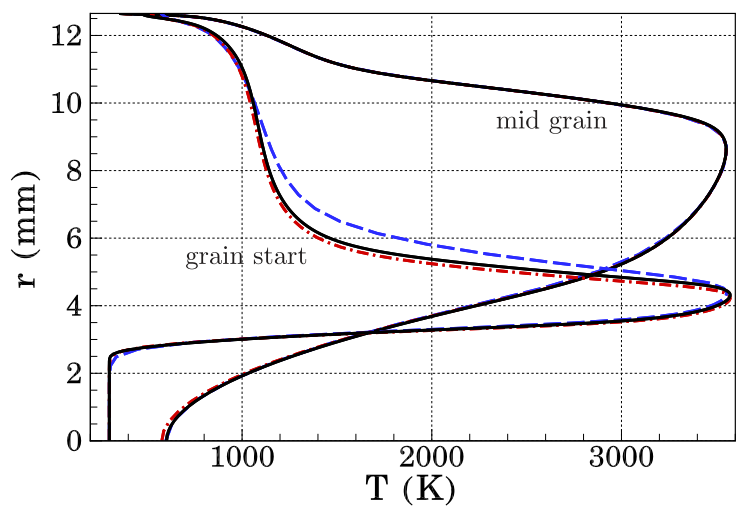

(d) Radial profiles of temperature at the start and in the middle of the grain.

Fig. 4 Grid sensitivity analysis performed on numerical simulations of test 4 (see Table 3).

and then moves away from the wall further downstream, because of the progressive consumption of the oxygen injected (Fig.6F). The flame reaches the centerline in proximity of the beginning of the nozzle because of the long post-chamber (note that $\left(x_{2}-x_{1}\right) / R \simeq 4.6$ ). Nevertheless, combustion reactants and products are inhomogeneously injected into the nozzle, where combustion still takes place. The average $\mathrm{O} / \mathrm{F}$ of test 4 is 1.17 , whereas the stoichiometric $\mathrm{O} / \mathrm{F}$ is 3.44 , indicating that the presence of fuel in the combustion chamber is predominant over the oxidizer. Even so, part of the injected oxygen directly flows away from the nozzle, reducing the combustion efficiency.

The pressure in the combustion chamber is approximately constant and reaches values close to the ones experimentally 


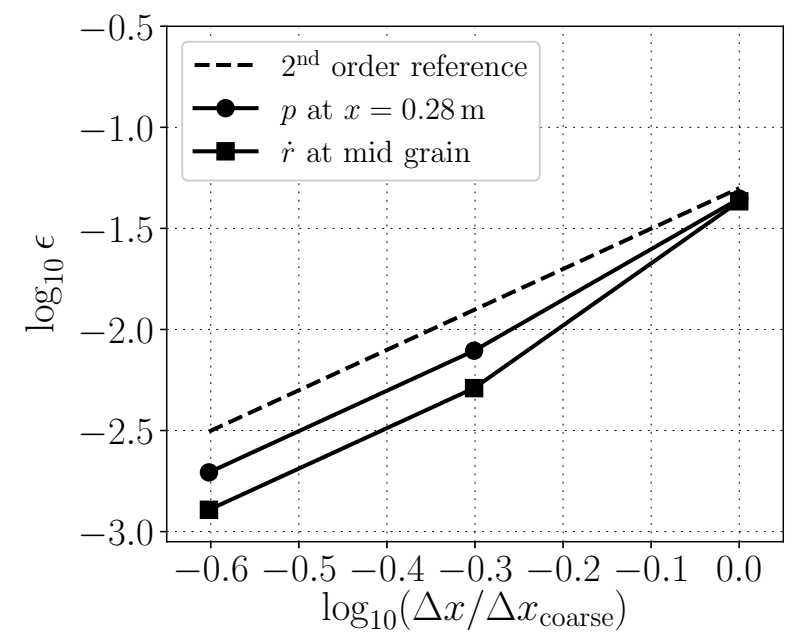

Fig. 5 Numerical error analysis performed on test 4 (see Table 3 ).

found (Fig. 77). The Mach number, while strongly dependent on the radial distance from the axis near the oxygen injection, where it develops from the almost null value of the recirculation region to approximately 0.3 in the pure oxygen stream, is eventually more uniform towards the end of the combustion chamber, where it is in the range $0.10-0.13$ (Fig.7p).

The mass fraction of the fuel available for combustion, which is made of molecular hydrogen and ethylene deriving from the thermal cracking of the paraffin-wax, is always lower than $8 \%$ (Fig. $8 \mathrm{a}, \mathrm{b}$ ). Molecular hydrogen is actually mostly present in a narrow layer off the wall. The fuels' combined mass fraction is highest in the pre- and post-chamber, with a considerable amount exiting the nozzle unburnt, consistent with the highly fuel-rich operating condition.

The species contributing to radiation $\left(\mathrm{H}_{2} \mathrm{O}, \mathrm{CO}\right.$, and $\left.\mathrm{CO}_{2}\right)$ are present from engine inlet to outlet (Fig. $\left.9 \mathrm{a}, \mathrm{b}, \mathrm{c}\right)$. However, most of the carbon monoxide is concentrated near the walls, while water vapor and carbon dioxide mass fractions are highest in correspondence of the highest flame temperature, but are also present close to the centerline. For all of them, a strong non-uniformity in the radial direction is observed. A large part of the flow covering the nozzle surface is composed by carbon monoxide and melted paraffin-wax, which is consistent with a close-to-null nozzle erosion [54, 68] as observed in the experiments [15].

The radial profiles appear smooth across the whole engine, confirming the effectiveness of the grid resolution employed: both the regions of the mixing layer (Fig. 10p) and of the combustion below the grain surface (Fig. 10p,c) are correctly reproduced. The peak temperature obtained is around $3450 \mathrm{~K}$, and its radial location coincides with the peak of carbon dioxide mass fraction, as already observed above. Near the peak of temperature, but closer to the fuel grain, the peak of carbon monoxide mass fraction is also found. Moreover, it is shown that the model is able to solve the wall mixture composition by accounting for the diffusion of all species towards the grain surface, where the melted paraffin-wax mass fraction is not equal to one. Away from the pre-chamber, the mass fraction of the melted paraffin-wax 


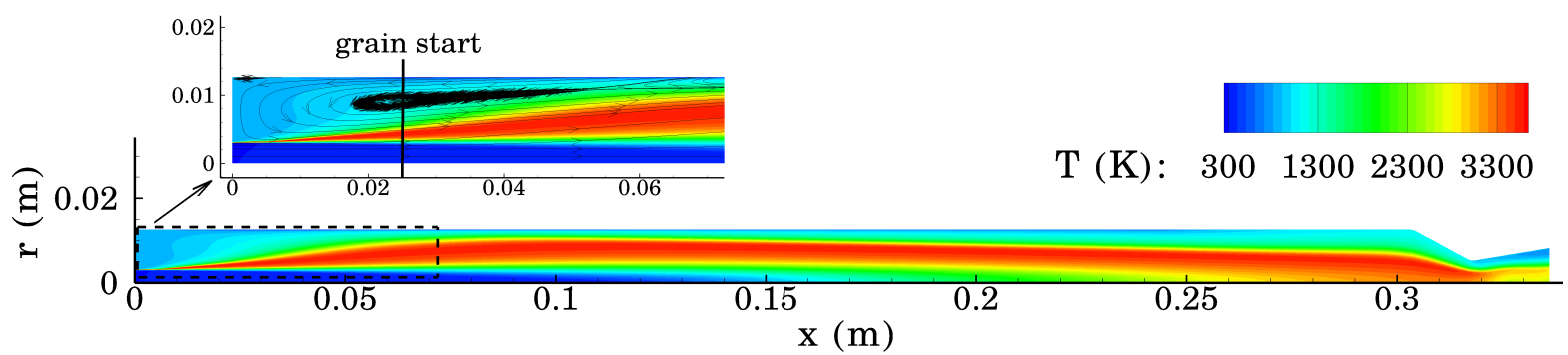

(a) Temperature contours with inset showing streamlines.

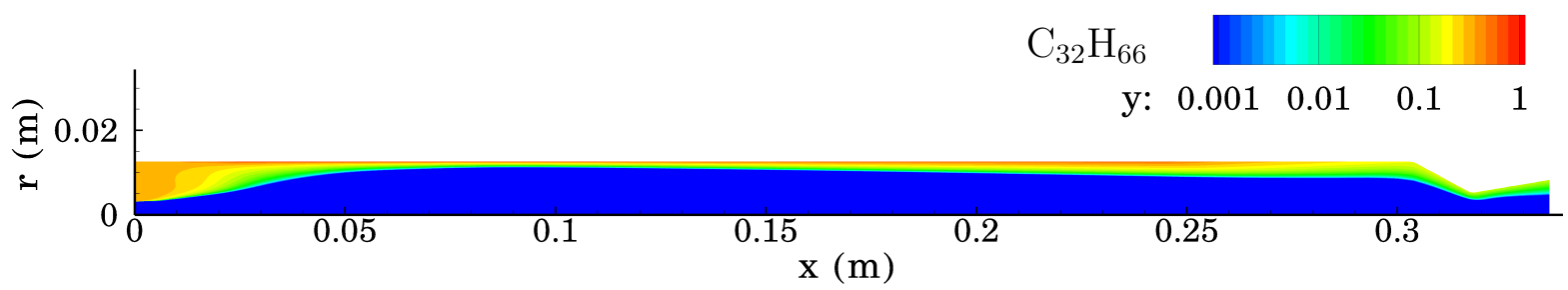

(b) Paraffin mass fraction contours.

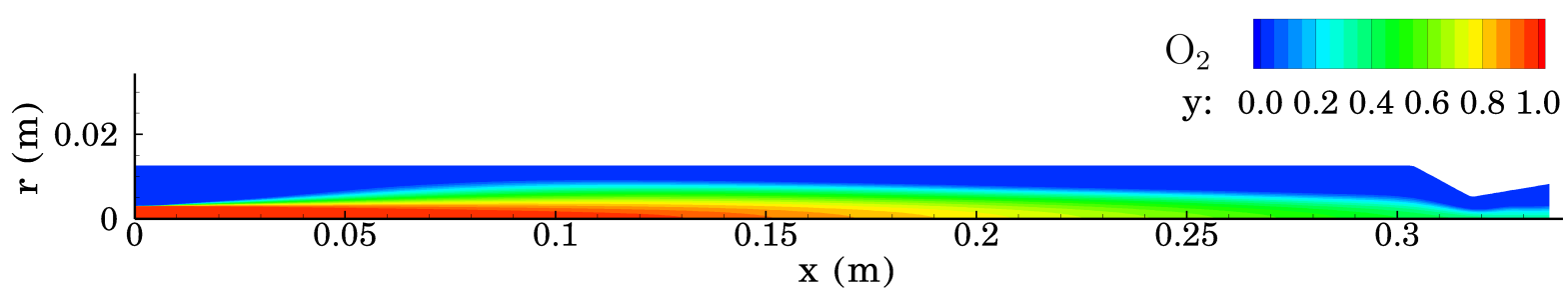

(c) Molecular oxygen mass fraction contours.

Fig. 6 Results on temperature, paraffin, and oxygen from numerical simulations of test 4.

decreases rapidly with increasing distance from the wall, due to its thermal cracking forming ethylene, which is however all burnt $3 \mathrm{~mm}$ below the wall. For paraffin mass fractions of $0.4,0.2$, and 0.1 , the fluid's temperature is approximately $800 \mathrm{~K}, 1000 \mathrm{~K}$, and $1200 \mathrm{~K}$, the last two of which are above paraffin-wax critical temperature $(860 \mathrm{~K})$, outside of the range of validity of the model for thermodynamic and transport properties adopted in this study (see section II.B.1. However for this test case paraffin-wax accounts for about $16 \%$ of the total fluid mass, with $53 \%$ of it at temperatures below $T_{c r}$, i.e. in the pressurized liquid state. Along the fuel grain $64 \%$ of the paraffin behaves like a pressurized liquid and it is mostly present inside of the cold boundary layer, close the where the actual energy exchange governing the regression rate takes place (Fig. 11], giving at least a fair confidence in the predictive capability of the present approach even if employing such a simplified thermodynamic model. 


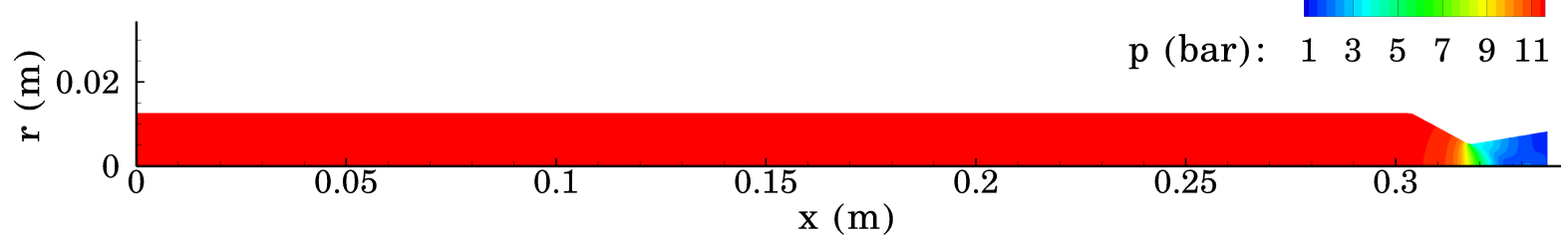

(a) Pressure contours.

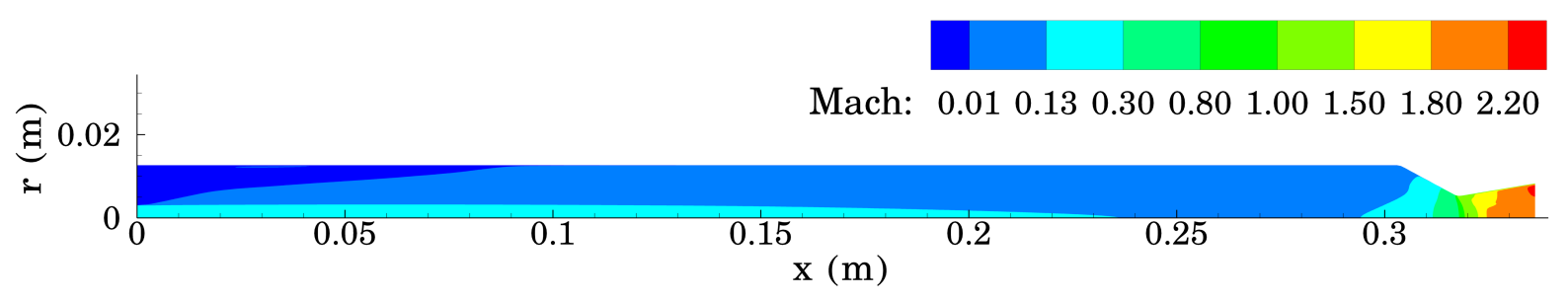

(b) Mach number contours.

Fig. 7 Results on pressure and Mach number from numerical simulations of test 4.

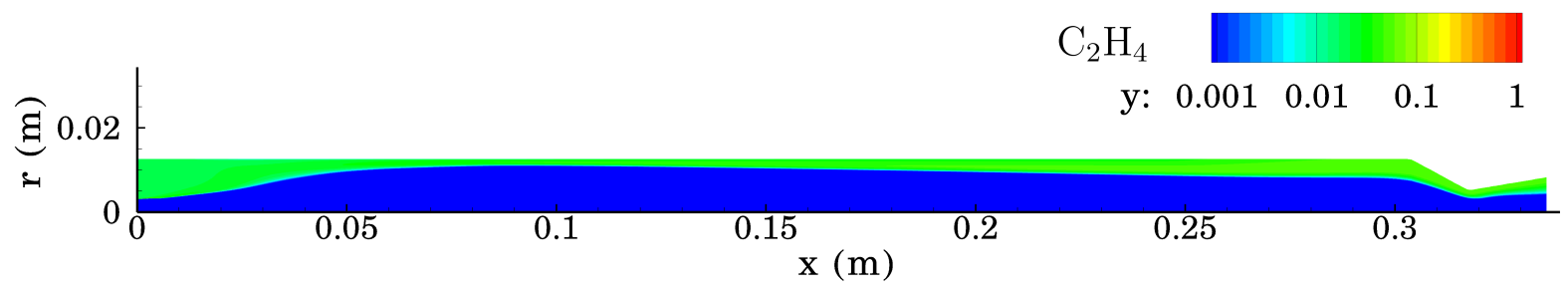

(a) Ethylene mass fraction contours.

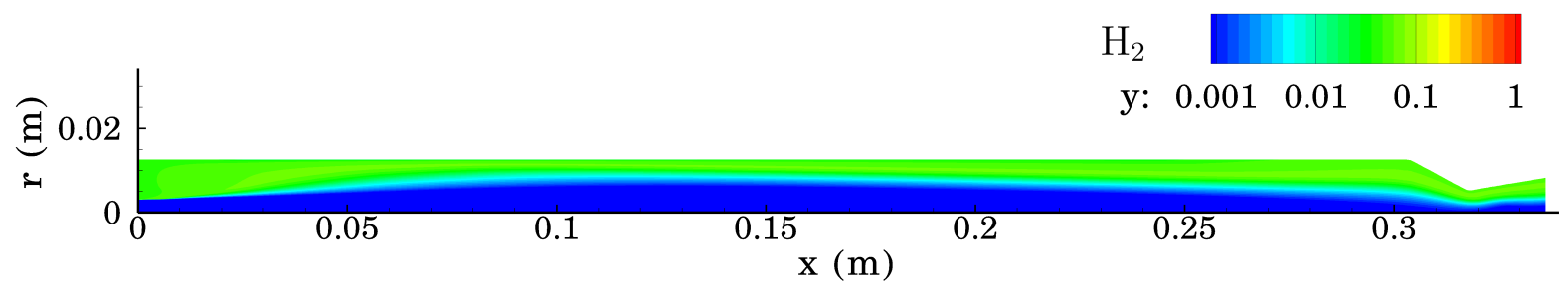

(b) Molecular hydrogen mass fraction contours.

Fig. 8 Results on the actual fuels burnt (see Table 1 from numerical simulations of test 4. 


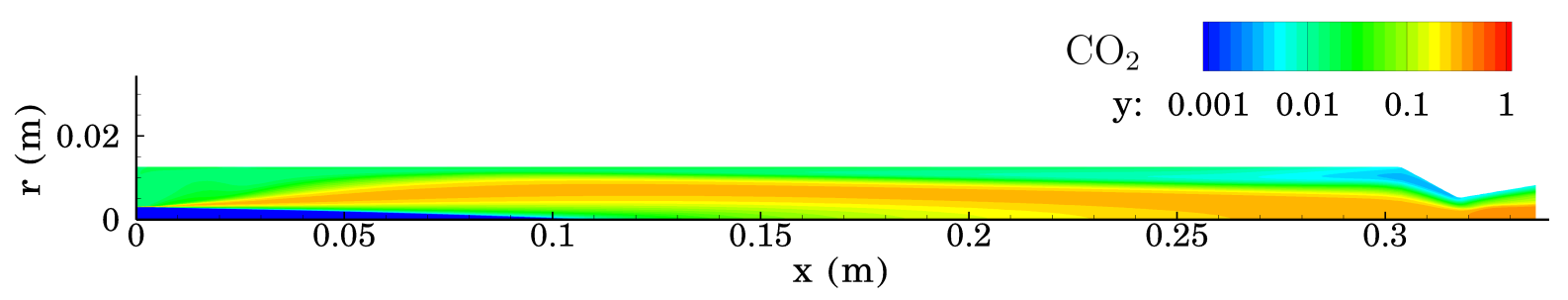

(a) Carbon dioxide mass fraction contours.

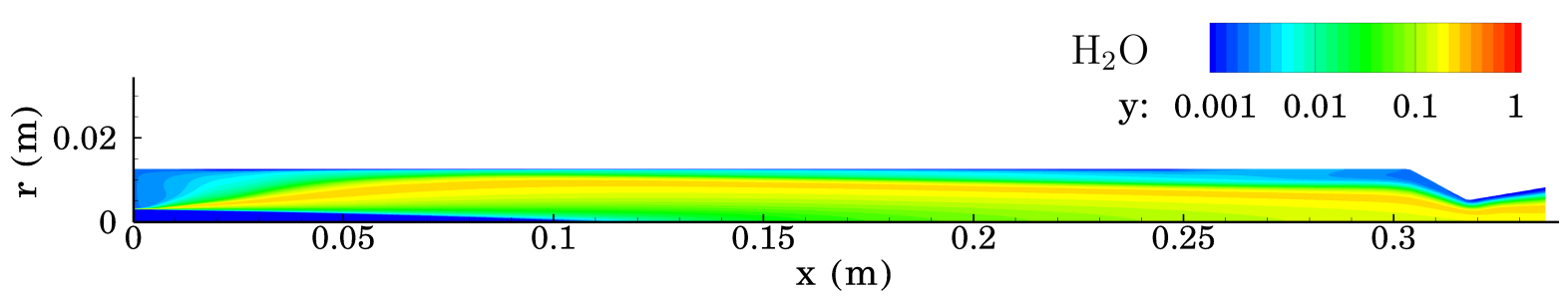

(b) Water mass fraction contours.

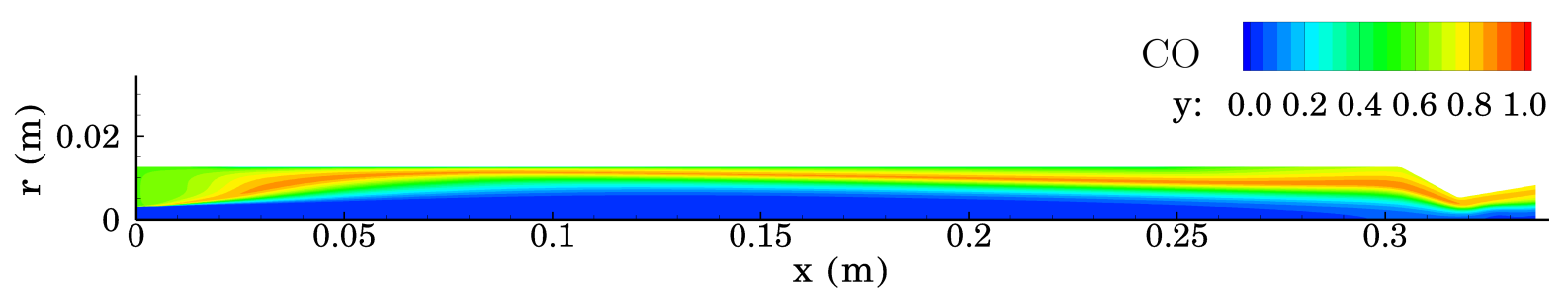

(c) Carbon monoxide mass fraction contours.

Fig. 9 Results on the radiating species from numerical simulations of test 4.

The fuel regression rate shows an inflection point at the location of flow reattachment (Fig. 12 $\mathrm{a}$ ), which moves further away from the injector for higher average port diameters. This behavior is due to the rather uniform convective heat flux on the grain above the recirculation region, which eventually grows steadily further downstream (Fig. [12p), causing the fuel injection to grow monotonically after reattachment, reaching its maximum at the end of the grain.

The most important effect obtained by increasing the size and the operating pressure of the engine is the larger radiative wall heat flux, which is observed to scale linearly with the product $p_{\mathrm{c}} R$ (Table 5, where $p_{\mathrm{c}}$ is the experimental chamber pressure) with a proportionality constant of 12.5 for this engine. Therefore, by assuming

$$
\bar{q}_{\mathrm{w}, \mathrm{rad}} \approx 12.5 p_{\mathrm{c}} R
$$




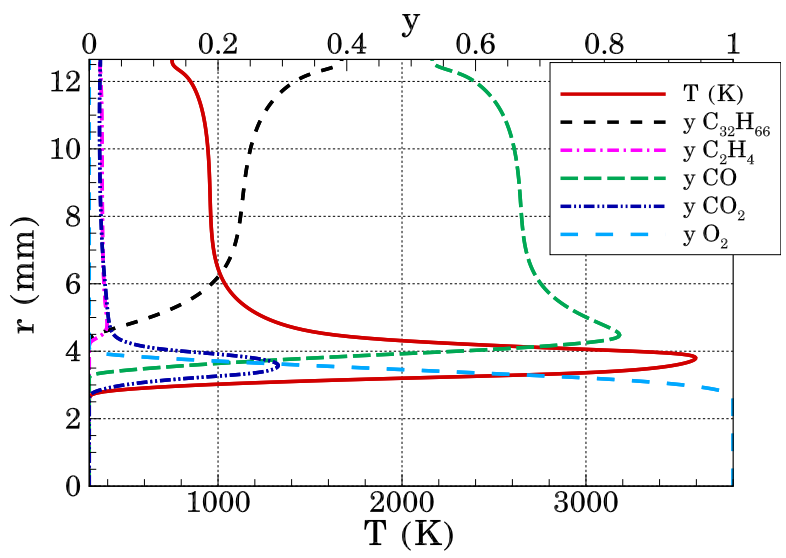

(a) Radial profiles extracted at $x=1.54 \mathrm{~cm}$.

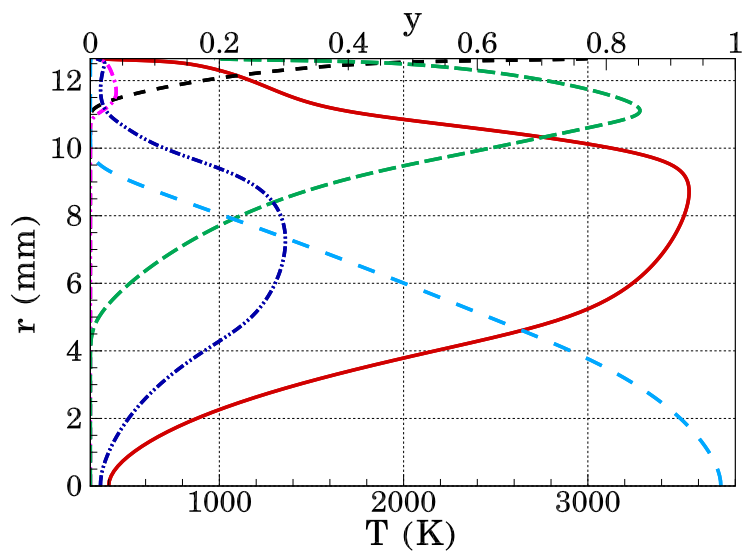

(b) Radial profiles extracted at $x=11.8 \mathrm{~cm}$.

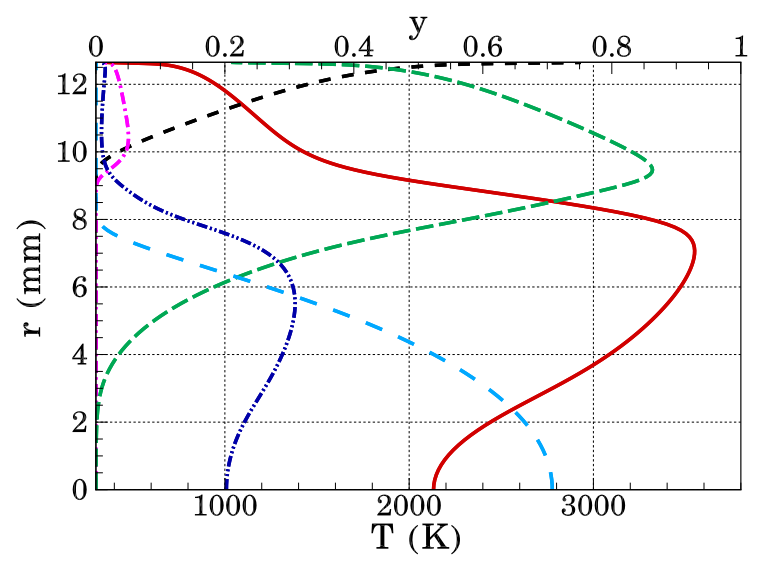

(c) Radial profiles extracted at $x=22.3 \mathrm{~cm}$.

Fig. 10 Radial profiles of temperature and mass fractions along the motor length for test 4 (see Table 3 .

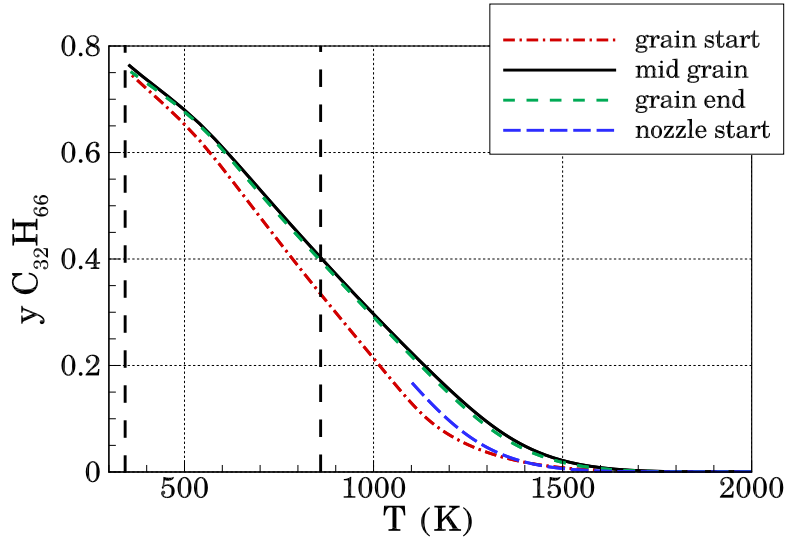

Fig. 11 Paraffin-wax mass fraction versus temperature at different axial locations for test 4 (see Table 3 . The vertical dashed lines indicate the melting and the critical temperature of paraffin-wax, respectively. 
Table 5 Wall heat flux contributions on the grain surface.

\begin{tabular}{cccccc}
\hline \hline test & $p_{\mathrm{c}} R($ bar $\times \mathrm{m})$ & $\bar{q}_{\mathrm{w}, \text { rad }}\left(\mathrm{MW} / \mathrm{m}^{2}\right)$ & $\bar{q}_{\mathrm{w}, \text { conv }}\left(\mathrm{MW} / \mathrm{m}^{2}\right)$ & $\bar{q}_{\mathrm{w}, \text { tot }}\left(\mathrm{MW} / \mathrm{m}^{2}\right)$ & $\bar{q}_{\mathrm{w}, \text { rad }} / \bar{q}_{\mathrm{w}, \text { tot }}(\%)$ \\
\hline 1 & 0.050 & 0.070 & 0.144 & 0.214 & 32.5 \\
3 & 0.100 & 0.131 & 0.249 & 0.380 & 34.6 \\
4 & 0.146 & 0.192 & 0.256 & 0.448 & 42.9 \\
$1 \mathrm{~W}$ & 0.175 & 0.220 & 0.223 & 0.443 & 49.7 \\
8 & 0.188 & 0.240 & 0.197 & 0.437 & 54.9 \\
9 & 0.228 & 0.277 & 0.203 & 0.480 & 57.7 \\
10 & 0.245 & 0.306 & 0.218 & 0.524 & 58.4 \\
12 & 0.258 & 0.322 & 0.256 & 0.578 & 55.7 \\
$2 \mathrm{~W}$ & 0.259 & 0.319 & 0.288 & 0.607 & 52.6 \\
11 & 0.281 & 0.337 & 0.206 & 0.543 & 62.1 \\
\hline \hline
\end{tabular}

and using Eq. (14) to obtain the expression for the total average heat flux $\bar{q}_{\mathrm{w}, \text { tot }}=\bar{q}_{\mathrm{w}, \mathrm{conv}}+\bar{q}_{\mathrm{w}, \mathrm{rad}}$,

$$
\bar{q}_{\mathrm{w}, \text { tot }}=\overline{\dot{r}} \rho_{\mathrm{s}} \Delta H
$$

where $\Delta H=\overline{\dot{r}} \rho_{\mathrm{s}}\left[\Delta h_{\text {melt }}+c_{\mathrm{s}}\left(T_{\text {melt }}-T_{\mathrm{s}, \text { in }}\right)\right]$ is a constant, an approximate expression for the relevance of radiation in the wall heat flux balance, which controls the mass flow rate of the injected paraffin and ranges from $33 \%$ to $62 \%$ in the cases considered in this study, can be obtained as

$$
\bar{q}_{\mathrm{w}, \mathrm{rad}} / \bar{q}_{\mathrm{w}, \mathrm{tot}} \approx 12.5 p_{\mathrm{c}} R /\left(\overline{\dot{r}} \rho_{\mathrm{s}} \Delta H\right)
$$

The axial profiles of the radiative heat flux are smooth in the whole combustion chamber (Fig. 12.), apart from the beginning and end of the grain where a cusp due to the sudden change from isothermal to adiabatic boundary conditions is observed. With increasing average radiative heat load, the location of its maximum moves downstream, but it is always present on the fuel grain. It is moreover noticed that radiation helps in generating a more uniform regression rate axial distribution.

\section{Experimental Rebuilding}

The integral average of the regression rate over the fuel surface is extracted from the numerical simulations for all cases of Table 3 and is compared to the respective experimental value in Fig. 13 a. The agreement between numerical and experimental regression rates $(<10-15 \%$ error $)$ is actually very good for these types of modeling approaches. However, notice that since the value of $\dot{r}$ is obtained indirectly from the fuel mass flux (see section II.B.2, a strong dependence on the fuel density at solid state exists. Indeed, the data in Fig. 13 are obtained with $\rho_{s}=920 \mathrm{~kg} / \mathrm{m}^{3}$, which is used 

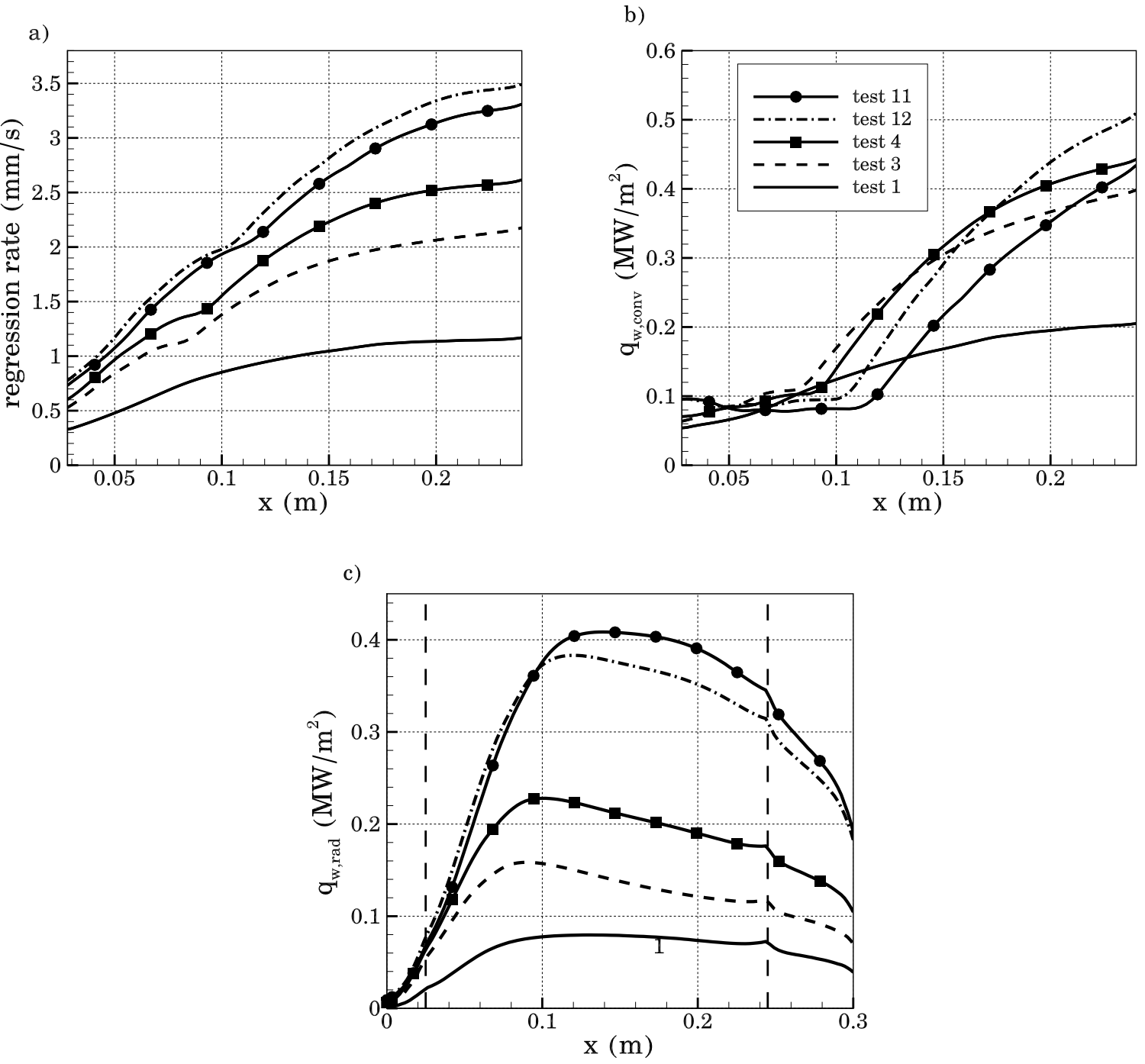

Fig. 12 Fuel regression rate (a), convective wall heat flux (b), and radiative wall heat flux (c) in the combustion chamber for some cases of Table 3 . The vertical dashed lines in (c) indicate the grain start and end, respectively. 
by [4, 15], while $830 \mathrm{~kg} / \mathrm{m}^{3}$ is used by [69] instead for a microcrystalline paraffin-wax with melting temperature of $346.15 \mathrm{~K}$, which is very close to the value adopted in this study (343 K).

By considering also the reported experimental errors (drawn as horizontal lines in Fig. 13a), the rebuilding can be considered accomplished with fair success. In test 1 the melted paraffin-wax is at subcritical pressure, which causes the large error obtained with the use of a single-phase numerical model if compared to the other supercritical tests. Moreover, tests $1 \mathrm{~W}$ and $2 \mathrm{~W}$ are the only ones that have been performed with a paraffin casted without the addition of a blackening additive: these are the tests for which the highest errors in regression rates are found among all supercritical cases. In particular, for test $1 \mathrm{~W}$ it is observed that the largest errors in the rebuilding of the axial profile of the fuel regression rates are present in correspondence of the vortex (Fig. 14). This can be due to the uneven axial consumption of fuel in time, not numerically captured by a single simulation at the average port diameter.

The wall pressure at $x=0.28 \mathrm{~m}$ obtained from the numerical simulations is found to be in excellent agreement with the respective time-averaged probe reading obtained during the firing tests (see Fig. 13b), interestingly, also for test 1 because of the large changes of $c^{*}$ at the computed $\mathrm{O} / \mathrm{F}$ (see below). A progressive weakening of the predictive accuracy is found for increasing chamber pressures, however leaving errors between the numerical predictions and the experimental values always below $10 \%$.
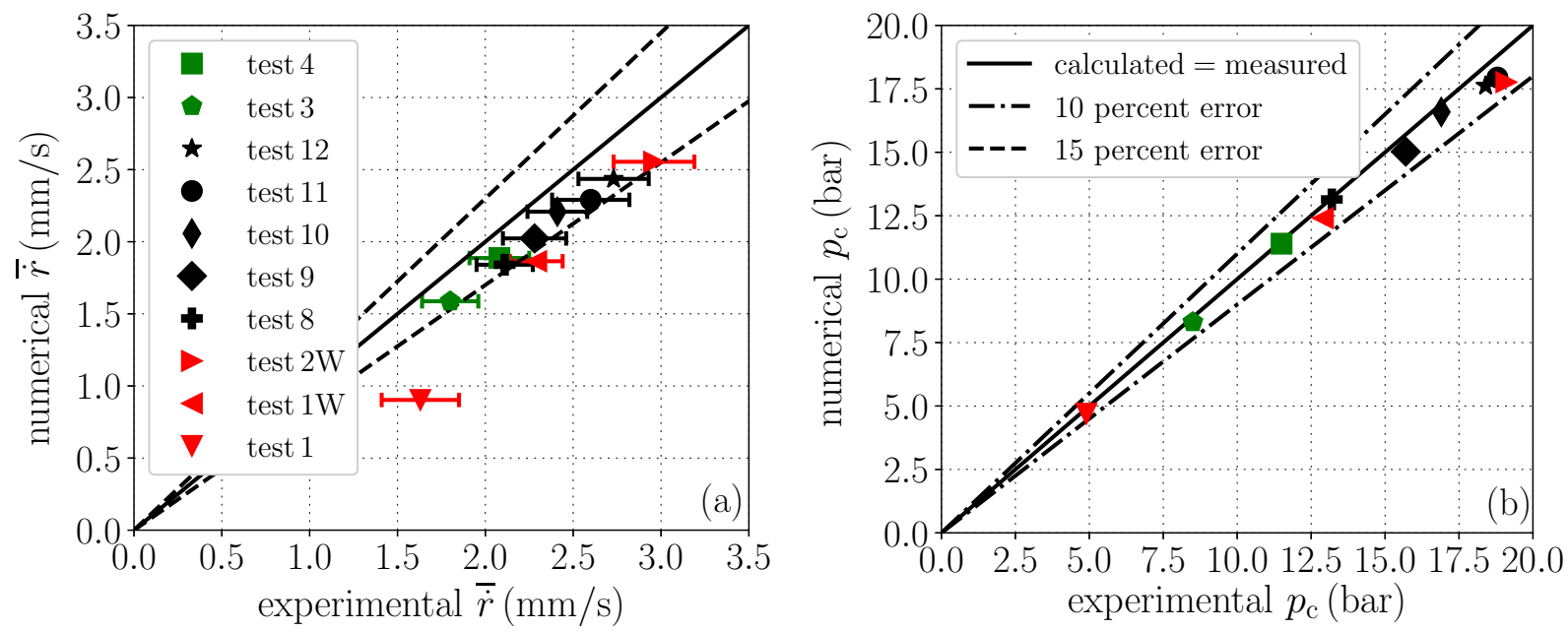

Fig. 13 (a) Experimental rebuilding of average regression rate with reported experimental uncertainties; (b) average postchamber pressure $(x=0.28 \mathrm{~m})$.

The characteristic velocity can be computed with

$$
c^{*}=\frac{p_{\mathrm{c}} A_{\mathrm{t}}}{\dot{m}_{\mathrm{ox}}} \frac{\mathrm{O} / \mathrm{F}}{1+\mathrm{O} / \mathrm{F}}
$$

where $A_{t}$ is the nozzle throat area. The combustion efficiency obtained with the experimentally measured average $\mathrm{O} / \mathrm{F}$ and pressure is generally slightly underestimated (by 1\% - 7\%) by the numerical computations (see Table 6), apart 


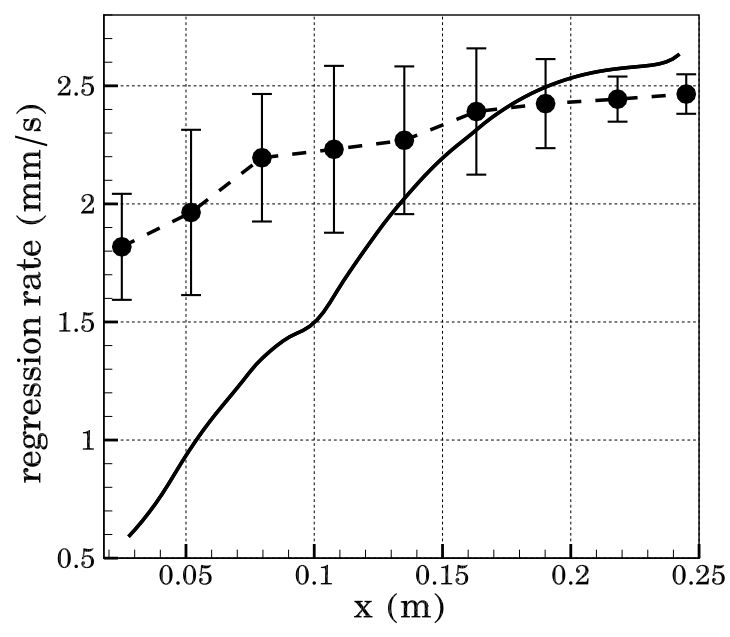

Fig. 14 Axial profile of fuel regression rate for test $1 \mathrm{~W}$ obtained with the numerical simulations (solid line) and experimental data with related uncertainty (full circles connected by dashed line).

from test 1 which is characterized by a subcritical chamber pressure, due to the simplified chemical model and to the geometrical simplifications employed. The O/F values are always overestimated, because of the lower values of numerical regression rates compared to the experimental ones. It is to be considered that the experimental $c^{*}$ efficiency is based upon time-averaged chamber pressure and $\mathrm{O} / \mathrm{F}$, and that the realistic experimental uncertainty in $c^{*}$ can be about $5 \%$ and the one in $\mathrm{O} / \mathrm{F}$ may reach $10 \%$ [70].

The numerical approach, which does not require information from any firing test, shows overall an acceptable prediction of the average experimental data, and only a slight under-prediction for regression rates, chamber pressures, and $c^{*}$ efficiency.

\section{Conclusions}

A numerical approach able to compute the internal ballistics of paraffin-based hybrid rocket engines has been developed and validated. The numerical method is based on the solution of the Reynolds-averaged Navier-Stokes equations, and includes sub-models for fluid-surface interaction, radiation, combustion, and turbulence. The main outcomes of the approach are the spatial profiles of the fuel regression rate and of the chamber pressure. Their average over the firing test has been compared successfully with ten recent experimental data in the literature, up to chamber pressures of 19.1 bar. The approach is not tuned to any specific firing test and is hence predictive.

Results show the dominating role of thermal radiation (up to $62 \%$ of the total heat flux) as the product of average port radius and average chamber pressure increases. For smaller port radius, for which radiation is less important, the model is still capable to fairly predict the correct amount of paraffin melted and injected in the combustion chamber, hence giving confidence to the obtained fluid-dynamic solution. 
Table 6 Experimental numerical oxidizer-to-fuel ratio, chamber pressure, theoretical characteristic velocity (computed with CEA at equilibrium conditions), and combustion efficiency.

\begin{tabular}{c|cccc|cccc}
\hline \hline & \multicolumn{6}{|c|}{ experimental } & \multicolumn{4}{c}{ numerical } \\
test & $\mathrm{O} / \mathrm{F}$ & $p_{\mathrm{c}}(\mathrm{bar})$ & $c_{\mathrm{th}}^{*}(\mathrm{~m} / \mathrm{s})$ & $\eta_{c^{*}}(\%)$ & $\mathrm{O} / \mathrm{F}$ & $p_{\mathrm{c}}(\mathrm{bar})$ & $c_{\mathrm{th}}^{*}(\mathrm{~m} / \mathrm{s})$ & $\eta_{c^{*}}(\%)$ \\
\hline 1 & 0.77 & 4.9 & 1426.5 & 82.42 & 1.36 & 4.70 & 1722.3 & 86.67 \\
3 & 1.04 & 8.5 & 1543.0 & 85.46 & 1.22 & 8.30 & 1639.4 & 84.64 \\
4 & 1.17 & 11.5 & 1605.0 & 87.41 & 1.28 & 11.4 & 1681.9 & 86.22 \\
8 & 1.16 & 13.2 & 1597.4 & 89.00 & 1.32 & 13.1 & 1701.6 & 88.11 \\
9 & 1.20 & 15.7 & 1627.0 & 92.53 & 1.35 & 15.03 & 1715.7 & 88.32 \\
10 & 1.26 & 16.9 & 1666.9 & 89.88 & 1.36 & 16.6 & 1724.3 & 88.23 \\
11 & 1.21 & 18.8 & 1634.0 & 92.65 & 1.38 & 17.95 & 1731.5 & 88.33 \\
12 & 1.19 & 18.4 & 1619.8 & 91.55 & 1.37 & 17.62 & 1728.9 & 87.45 \\
$1 \mathrm{~W}$ & 1.08 & 12.9 & 1559.3 & 90.26 & 1.31 & 12.41 & 1694.8 & 87.13 \\
$2 \mathrm{~W}$ & 1.20 & 19.1 & 1627.0 & 93.40 & 1.37 & 17.76 & 1730.0 & 86.70 \\
\hline \hline
\end{tabular}

The numerical approach provides the capability of internal ballistics prediction and paves the way to novel designoriented investigations for paraffin-based hybrid rockets. Future work will include comparison of the numerical results with more experimental data, including the extension of the investigated pressure and $\mathrm{O} / \mathrm{F}$ ranges, and scale effects. Furthermore, improvements on the sub-models employed, such as considering global kinetic mechanisms more relevant to the combustion of ethylene, or multi-component diffusion models, are foreseen.

\section{Acknowledgments}

This study was partially supported by the Italian Space Agency under framework agreement number 2018-34-HH.0, and by the Italian Ministry of Education, University, and Research.

\section{References}

[1] Altman, D., and Holzman, A., "Overview and History of Hybrid Rocket Propulsion," Fundamentals of Hybrid Rocket Combustion and Propulsion, Progress in Astronautics and Aeronautics, Vol. 218, edited by K. K. Kuo and M. J. Chiaverini, AIAA, 2007, pp. 1-36. doi:10.2514/5.9781600866876.0001.0036.

[2] Cantwell, B. J., Karabeyoglu, M. A., and Altman, D., "Recent Advances in Hybrid Propulsion,” International Journal of Energetic Materials and Chemical Propulsion, Vol. 9, No. 4, 2010, pp. 305-326. doi:10.1615/IntJEnergeticMaterialsChemProp.v9.i4.20.

[3] Karp, A. C., Nakazono, B., Shotwell, R., Benito, J., Vaughan, D., and Story, G. T., "Technology Development Plan and Preliminary Results for a Low Temperature Hybrid Mars Ascent Vehicle Concept,” AIAA Paper 2017-4900, Jul. 2017. doi:10.2514/6.2017-4900. 
[4] Karabeyoglu, M. A., Altman, D., and Cantwell, B. J., "Combustion of Liquefying Hybrid Propellants: Part 1, General Theory," Journal of Propulsion and Power, Vol. 18, No. 3, 2002, pp. 610-620. doi:10.2514/2.5975.

[5] Karabeyoglu, M. A., and Cantwell, B. J., "Combustion of Liquefying Hybrid Propellants: Part 2, Stability of Liquid Films," Journal of Propulsion and Power, Vol. 18, No. 3, 2002, pp. 621-630. doi:10.2514/2.5976.

[6] Karabeyoglu, M. A., Cantwell, B. J., and Altman, D., "Development and Testing of Paraffin-based Hybrid Rocket Fuels," AIAA Paper 2001-4503, Aug. 2001. doi:10.2514/6.2001-4503.

[7] Karabeyoglu, M. A., Zilliac, G., Cantwell, B. J., DeZilwa, S., and Castellucci, P., "Scale-Up Tests of High Regression Rate Paraffin-Based Hybrid Rocket Fuels,” Journal of Propulsion and Power, Vol. 20, No. 6, 2004, pp. 1037-1045. doi: $10.2514 / 1.3340$.

[8] Marxman, G. A., Wooldridge, C. E., and Muzzy, R. J., "Fundamentals of Hybrid Boundary-Layer Combustion," Heterogeneous Combustion, Progress in Astronautics and Rocketry, Vol. 15, edited by H. G. Wolfhard, I. Glassman, and L. Green, Elsevier, 1964, pp. 485-522. doi:10.1016/B978-1-4832-2730-6.50025-7.

[9] Leccese, G., Cavallini, E., and Pizzarelli, M., "State of Art and Current Challenges of the Paraffin-Based Hybrid Rocket Technology,” AIAA Paper 2019-4010, Aug. 2019. doi:10.2514/6.2019-4010.

[10] Adachi, M., and Shimada, T., "Liquid Films Instability Analysis of Liquefying Hybrid Rocket Fuels Under Supercritical Conditions,” AIAA Journal, Vol. 53, No. 6, 2015, pp. 1578-1589. doi:10.2514/1.J053459.

[11] Bellomo, N., Barato, F., Faenza, M., Lazzarin, M., Bettella, A., and Pavarin, D., "Numerical and Experimental Investigation of Unidirectional Vortex Injection in Hybrid Rocket Engines,” Journal of Propulsion and Power, Vol. 29, No. 5, 2013, pp. 1097-1113. doi:10.2514/1.B34506.

[12] Ranuzzi, G., Cardillo, D., and Invigorito, M., "Numerical Investigation of a $\mathrm{N}_{2} \mathrm{O}-$ Paraffin Hybrid Rocket Engine Combusting Flowfield," $\sigma^{\text {th }}$ European Conference for Aeronautics and Space Sciences (EUCASS), Krakow, Poland, 2015. doi:10.13140/RG. 2.1.1329.6727.

[13] Lazzarin, M., Faenza, M., Barato, F., Bellomo, N., Bettella, A., and Pavarin, D., "Computational Fluid Dynamics Simulation of Hybrid Rockets of Different Scales," Journal of Propulsion and Power, Vol. 31, No. 5, 2015, pp. 1458-1469. doi: 10.2514/1.B35528.

[14] Paccagnella, E., Barato, F., Gelain, R., and Pavarin, D., "CFD Simulations of Self-pressurized Nitrous Oxide Hybrid Rocket Motors,” AIAA Paper 2018-4534, Jul. 2018. doi:10.2514/6.2018-4534.

[15] Di Martino, G. D., Mungiguerra, S., Carmicino, C., Savino, R., Cardillo, D., Battista, F., Invigorito, M., and Elia, G., “Two-Hundred-Newton Laboratory-Scale Hybrid Rocket Testing for Paraffin Fuel-Performance Characterization,” Journal of Propulsion and Power, Vol. 35, No. 1, 2019, pp. 224-235. doi:10.2514/1.B37017. 
[16] Bianchi, D., Nasuti, F., and Delfini, D., "Modeling of Gas-surface Interface for Paraffin-based Hybrid Rocket Fuels in Computational Fluid Dynamics Simulations," Progress in Propulsion Physics, Vol. 11, 2019, pp. 3-24. doi:10.1051/eucass/ 201911003.

[17] Di Martino, G. D., Mungiguerra, S., Carmicino, C., and Savino, R., "Computational Fluid-dynamic Modeling of the Internal Ballistics of Paraffin-fueled Hybrid Rocket,” Aerospace Science and Technology, Vol. 89, 2019 , pp. 431 - 444. doi:10.1016/j.ast.2019.04.019.

[18] Chiaverini, M. J., "Review of Solid-Fuel Regression Rate Behavior in Classical and Nonclassical Hybrid Rocket Motors," Fundamentals of Hybrid Rocket Combustion and Propulsion, Progress in Astronautics and Aeronautics, Vol. 218, edited by K. K. Kuo and M. J. Chiaverini, AIAA, 2007, pp. 37-126. doi:10.2514/5.9781600866876.0037.0126.

[19] Bianchi, D., Leccese, G., Nasuti, F., and Carmicino, C., "Modeling of High Density Polyethylene Regression Rate in the Simulation of Hybrid Rocket Flowfields," $7^{\text {th }}$ European Conference for Aeronautics and Space Sciences (EUCASS), Milan, Italy, 2017. doi:10.13009/EUCASS2017-629.

[20] Durand, J.-E., Raynaud, F., Lamet, J.-M., Tessé, L., Lestrade, J.-Y., and Anthoine, J., "Numerical Study of Fuel Regression in Hybrid Rocket Engine,” AIAA Paper 2018-4593, Jul. 2018. doi:10.2514/6.2018-4593.

[21] Leccese, G., Bianchi, D., and Nasuti, F., “Modeling and Simulation of Paraffin-Based Hybrid Rocket Internal Ballistics,” AIAA Paper 2018-4533, Jul. 2018. doi:10.2514/6.2018-4533.

[22] Leccese, G., Bianchi, D., and Nasuti, F., "Numerical Investigation on Radiative Heat Loads in Liquid Rocket Thrust Chambers," Journal of Propulsion and Power, Vol. 35, No. 5, 2019, pp. 930-943. doi:10.2514/1.B37536.

[23] Migliorino, M. T., Bianchi, D., and Nasuti, F., "Predictive CFD Model for Internal Ballistics of Hybrid Rocket Engines using Supercritical Paraffin-wax and Oxygen,” AIAA Paper 2019-4261, Aug. 2019. doi:10.2514/6.2019-4261.

[24] Chandler, A. A., Jens, E. T., Cantwell, B. J., and Hubbard, G. S., "Visualization of the liquid layer combustion of paraffin fuel at elevated pressures," 63 ${ }^{\text {rd }}$ International Astronautical Congress, 2012.

[25] Petrarolo, A., Kobald, M., and Schlechtriem, S., "Visualization of Combustion Phenomena in Paraffin-Based Hybrid Rocket Fuels at Super-Critical Pressures," AIAA Paper 2018-4927, 2018. doi:10.2514/6.2018-4927.

[26] Anderson, J. D., Hypersonic and High-Temperature Gas Dynamics, $2^{\text {nd }}$ ed., AIAA Education series, Reston, VA, 2006, pp. 596-617.

[27] Gordon, S., and McBride, B. J., "Computer Program for Calculation of Complex Chemical Equilibrium Compositions and Applications. I. Analysis,” NASA RP-1311, Oct. 1994.

[28] Spalart, P. R., and Allmaras, S. R., “A One-Equation Turbulence Model for Aerodynamic Flows,” La Recherche Aerospatiale, Vol. 1, 1994, pp. 5-21. 
[29] McBride, B. J., Zehe, M. J., and Gordon, S., "NASA Glenn Coefficients for Calculating Thermodynamic Properties of Individual Species," Tech. Rep. NASA/TP-2002-211556, Sep. 2002.

[30] Coronetti, A., and Sirignano, W. A., "Numerical Analysis of Hybrid Rocket Combustion," Journal of Propulsion and Power, Vol. 29, No. 2, 2013, pp. 371-384. doi:10.2514/1.B34760.

[31] Fabuss, B. M., Smith, J. O., Lait, R. I., Bornsanyi, A. S., and Satterfield, C. N., "Rapid Thermal Cracking of n-Hexadecane at Elevated Pressures," Industrial \& Engineering Chemistry Process Design and Development, Vol. 1, No. 4, 1962, pp. $293-299$. doi:10.1021/i260004a011.

[32] Blouri, B., Hamdan, F., and Herault, D., "Mild Cracking of High-Molecular-Weigth Hydrocarbons," Industrial \& Engineering Chemistry Process Design and Development, Vol. 1, No. 24, 1985, pp. 30-37. doi:10.1021/i200028a005.

[33] Betti, B., Bianchi, D., Nasuti, F., and Martelli, E., "Chemical Reaction Effects on Heat Loads of CH4/O2 and H2/O2 Rockets," AIAA Journal, Vol. 54, No. 5, 2016, pp. 1693-1703. doi:10.2514/1.J054606.

[34] Betti, B., Martelli, E., Nasuti, F., and Onofri, M., "Numerical Study of Heat Transfer in Film Cooled Thrust Chambers," AIAA Paper 2012-3907, Jul. 2012. doi:10.2514/6.2012-3907.

[35] Pizzarelli, M., Nasuti, F., Paciorri, R., and Onofri, M., "Numerical Analysis of Three-Dimensional Flow of Supercritical Fluid in Cooling Channels," AIAA Journal, Vol. 47, No. 11, 2009, pp. 2534-2543. doi:10.2514/1.38542.

[36] Betti, B., Nasuti, F., and Martelli, E., "Numerical Evaluation of Heat Transfer Enhancement in Rocket Thrust Chambers by Wall Ribs," Numerical Heat Transfer Part A: Applications, Vol. 66, No. 5, 2014, pp. 488-508. doi:10.1080/10407782.2014.885233.

[37] Betti, B., Pizzarelli, M., and Nasuti, F., "Coupled Heat Transfer Analysis in Regeneratively Cooled Thrust Chambers,” Journal of Propulsion and Power, Vol. 30, No. 2, 2014, pp. 360-367. doi:10.2514/1.B34855.

[38] Bianchi, D., Betti, B., Nasuti, F., and Carmicino, C., "Simulation of Gaseous Oxygen/Hydroxyl-Terminated Polybutadiene Hybrid Rocket Flowfields and Comparison with Experiments," Journal of Propulsion and Power, Vol. 31, No. 3, 2015 , pp. 919-929. doi:10.2514/1.B35587.

[39] Bianchi, D., Nasuti, F., and Carmicino, C., "Hybrid Rockets with Axial Injector: Port Diameter Effect on Fuel Regression Rate," Journal of Propulsion and Power, Vol. 32, No. 4, 2016, pp. 984-996. doi:10.2514/1.B36000.

[40] Roe, P. L., “Approximate Riemann Solvers, Parameter Vectors and Difference Schemes,” Journal of Computational Physics, Vol. 43, 1981, pp. 357-372. doi:10.1016/0021-9991(81)90128-5.

[41] Strang, G., "On the Construction and Comparison of Difference Schemes," SIAM Journal on Numerical Analysis, Vol. 5, 1968, pp. 506-517. doi:10.1137/0705041.

[42] Brown, P. N., Byrne, G. D., and Hindmarsh, A. C., "VODE: A Variable-Coefficient ODE Solver," SIAM Journal on Scientific and Statistical Computing, Vol. 10, No. 5, 1989, pp. 1038-1051. doi:10.1137/0910062. 
[43] Linstrom, P. J., and Mallard, W. G., NIST Chemistry WebBook, NIST Standard Reference Database Number 69, National Institute of Standards and Technology, Gaithersburg MD, 20899, 2019. doi:10.18434/T4D303.

[44] Marano, J. J., and Holder, G. D., "General Equation for Correlating the Thermophysical Properties of n-Paraffins, n-Olefins, and Other Homologous Series. 2. Asymptotic Behavior Correlations for PVT Properties," Industrial \& Engineering Chemistry Research, Vol. 36, No. 5, 1997, pp. 1895-1907. doi:10.1021/ie960512f.

[45] Oschwald, M., and Schik, A., "Supercritical Nitrogen Free Jet Investigated by Spontaneous Raman Scattering," Experiments in Fluids, Vol. 27, No. 6, 1999, pp. 497-506. doi:10.1007/s003480050374.

[46] Oschwald, M., and Micci, M., "Spreading Angle and Centerline Variation of Density of Supercritical Nitrogen Jets," Atomization and Sprays, Vol. 12, No. 1-3, 2002, pp. 91-106.

[47] Marano, J. J., and Holder, G. D., "General Equation for Correlating the Thermophysical Properties of n-Paraffins, n-Olefins, and Other Homologous Series. 3. Asymptotic Behavior Correlations for Thermal and Transport Properties," Industrial \& Engineering Chemistry Research, Vol. 36, No. 6, 1997, pp. 2399-2408. doi:10.1021/ie9605138.

[48] Quan, V., "Quasi-Steady Solution for Ablation-Erosion Heat Transfer," Journal of Spacecraft and Rockets, Vol. 7, No. 3, 1970, pp. 355-357. doi:10.2514/3.29938.

[49] Karabeyoglu, M. A., Cantwell, B. J., and Stevens, J., "Evaluation of the Homologous Series of Normal Alkanes as Hybrid Rocket Fuels,” AIAA Paper 2005-3908, 2005. doi:10.2514/6.2005-3908.

[50] Baer, D., and Ambrosio, A., "Heat conduction in a semi-infinite slab with sublimation at the surface," Planetary and Space Science, Vol. 4, 1961, pp. 436 - 446. doi:10.1016/0032-0633(61)90150-7.

[51] Flood, D. T., Kendall, R. M., and Rindal, R. A., "Analytical and experimental study of ablation material for rocket engine application,” NASA CR-54757, May 1996.

[52] Bianchi, D., Nasuti, F., Paciorri, R., and Onofri, M., “Computational Analysis of Hypersonic Flows Including Finite Rate Ablation Thermochemistry," $7^{\text {th }}$ European Workshop on Thermal Protection Systems and Hot Structures, ESA-ESTEC, Noordwijk, The Netherlands, 2013.

[53] Turchi, A., Bianchi, D., Nasuti, F., and Onofri, M., "A Numerical Approach for the Study of the Gas-surface Interaction in Carbon-phenolic Solid Rocket Nozzles,” Aerospace Science and Technology, Vol. 27, No. 1, 2013, pp. 25-31. doi: 10.1016/j.ast.2012.06.003.

[54] Bianchi, D., and Nasuti, F., "Numerical Analysis of Nozzle Material Thermochemical Erosion in Hybrid Rocket Engines," Journal of Propulsion and Power, Vol. 29, No. 3, 2013, pp. 547-558. doi:10.2514/1.B34813.

[55] Bianchi, D., Betti, B., Nasuti, F., and Carmicino, C., "Simulation of Gaseous Oxygen/Hydroxyl-Terminated Polybutadiene Hybrid Rocket Flowfields and Comparison with Experiments," Journal of Propulsion and Power, Vol. 31, No. 3, 2015 , pp. 919-929. doi:10.2514/1.B35587. 
[56] Bianchi, D., Leccese, G., Nasuti, F., Onofri, M., and Carmicino, C., "Modeling of High Density Polyethylene Regression Rate in the Simulation of Hybrid Rocket Flowfields," Aerospace, Vol. 6, No. 8, 2019. doi:10.3390/aerospace6080088.

[57] Leccese, G., Bianchi, D., Betti, B., Lentini, D., and Nasuti, F., "Convective and Radiative Wall Heat Transfer in Liquid Rocket Thrust Chambers," Journal of Propulsion and Power, Vol. 34, No. 2, 2018, pp. 318-326. doi:10.2514/1.B36589.

[58] Leccese, G., Bianchi, D., Nasuti, F., Stober, K. J., Narsai, P., and Cantwell, B. J., "Experimental and Numerical Methods for Radiative Wall Heat Flux Predictions in Paraffin-based Hybrid Rocket Engines,” Acta Astronautica, Vol. 158, 2019, pp. 304 312. doi:10.1016/j.actaastro.2018.06.032.

[59] Hsu, S. T., Engineering Heat Transfer, D. Van Nostrand Company, Inc., Princeton, 1963.

[60] Liu, J., and Tiwari, S. N., "Radiative Heat Transfer Effects in Chemically Reacting Nozzle Flows," Journal of Thermophysics and Heat Transfer, Vol. 10, No. 3, 1996, pp. 436-444. doi:10.2514/3.808.

[61] Modest, M. F., Radiative Heat Transfer, Academic Press, San Diego, 2013, pp. 288-410.

[62] Tien, C. L., "Thermal Radiation Properties of Gases," Advances in Heat Transfer, Vol. 5, edited by T. F. Irvine and J. P. Hartnett, Elsevier, 1969, pp. 253-324. doi:10.1016/S0065-2717(08)70131-X.

[63] Rivière, P., and Soufiani, A., "Updated Band Model Parameters for $\mathrm{H}_{2} \mathrm{O}, \mathrm{CO}_{2}, \mathrm{CH}_{4}$ and $\mathrm{CO}$ Radiation at High Temperature," International Journal of Heat and Mass Transfer, Vol. 55, No. 13, 2012, pp. 3349-3358. doi:10.1016/j.ijheatmasstransfer.2012. 03.019 .

[64] Chmielewski, M., and Gieras, M., "Planck Mean Absorption Coefficients of $\mathrm{H}_{2} \mathrm{O}, \mathrm{CO}_{2}, \mathrm{CO}$ and NO for radiation numerical modeling in combusting flows," Journal of Power Technologies, Vol. 95, No. 2, 2015, pp. 97-104.

[65] Astarita, T., and Carlomagno, G. M., Infrared Thermography for Thermo-Fluid-Dynamics, Springer-Verlag Berlin Heidelberg, 2013. doi:10.1007/978-3-642-29508-9.

[66] Roache, P. J., "Verification of Codes and Calculations," AIAA Journal, Vol. 36, No. 5, 1998, pp. 696-702. doi:10.2514/2.457.

[67] Bonfiglioli, A., and Paciorri, R., "Convergence Analysis of Shock-Capturing and Shock-Fitting Solutions on Unstructured Grids,” AIAA Journal, Vol. 52, No. 7, 2014, pp. 1404-1416. doi:10.2514/1.J052567.

[68] Bianchi, D., Migliorino, M. T., Nasuti, F., and Onofri, M., "CFD Analysis of Paraffin-Based Hybrid Rockets with Coupled Nozzle Erosion Characterization,” AIAA Paper 2019-4263, Aug. 2019. doi:10.2514/6.2019-4263.

[69] Freund, M., Csikos, R., Keszthelyi, S., and Mozes, G., Paraffin Products: Properties, Technologies, Applications, Elsevier Scientific Publishing Company, Amsterdam, 1982.

[70] Weinstein, A., and Gany, A., “Testing and modeling liquefying fuel combustion in hybrid propulsion," Progress in Propulsion Physics, Vol. 4, 2013, pp. 99-112. doi:10.1051/eucass/201304099. 